\title{
The Dense Molecular Gas and Nuclear Activity in the ULIRG IRAS 13120-5453
}

\author{
G. C. Privon ${ }^{1,2}$, S. Aalto ${ }^{3}$, N. Falstad ${ }^{3}$, S. Muller ${ }^{3}$, E. González-Alfonso ${ }^{4}$, K. Sliwa ${ }^{5}$, E. Treister ${ }^{1,2}$, F. Costagliola ${ }^{3}$ L. Armus $^{6}$, \\ A. S. Evans ${ }^{7,8}$, S. Garcia-Burillo ${ }^{9}$, T. Izumi ${ }^{10}$, K. Sakamoto ${ }^{11}$, P. van der Werf ${ }^{12}$, and J. K. Chu ${ }^{13}$ \\ ${ }^{1}$ Instituto de Astrofśica, Facultad de Física, Pontificia Universidad Católica de Chile, Casilla 306, Santiago 22, Chile \\ ${ }^{2}$ Departamento de Astronomía, Universidad de Concepción, Casilla 160-C, Concepción, Chile \\ ${ }^{3}$ Department of Earth and Space Sciences, Chalmers University of Technology, Onsala Space Observatory, SE-439 94 Onsala, Sweden \\ ${ }^{4}$ Universidad de Alcalá, Departamento de Física y Matemáticas, Campus Universitario, E-28871 Alcalá de Henares, Madrid, Spain \\ ${ }^{5}$ Max Planck Institute for Astronomy, Königstuhl 17, D-69117 Heidelberg, Germany \\ ${ }^{6}$ Spitzer Science Center, California Institute of Technology, MS 220-6, Pasadena, CA, 91125, USA \\ ${ }^{7}$ Department of Astronomy, University of Virginia, Charlottesville, VA 22903, USA \\ ${ }_{8}^{8}$ National Radio Astronomy Observatory, Charlottesville, VA, 22903 USA \\ ${ }^{9}$ Observatorio de Madrid, OAN-IGN, Alfonso XII, 3, E-28014-Madrid, Spain \\ ${ }^{10}$ Institute of Astronomy, School of Science, The University of Tokyo, 2-21-1 Osawa, Mitaka, Tokyo 181-0015, Japan \\ ${ }^{11}$ Institute of Astronomy and Astrophysics, Academia Sinica, P.O. Box 23-141, 10617, Taipei, Taiwan \\ ${ }^{12}$ Leiden Observatory, Leiden University, P.O. Box 9513, 2300 RA Leiden, The Netherlands \\ ${ }^{13}$ Institute for Astronomy, University of Hawaii, 2680 Woodlawn Drive, Honolulu, HI 96822, USA \\ Received 2016 September 9; revised 2016 November 29; accepted 2016 December 8; published 2017 January 31
}

\begin{abstract}
We present new Atacama Large Millimeter/submillimeter Array Band $7(\sim 340 \mathrm{GHz})$ observations of the dense gas tracers $\mathrm{HCN}, \mathrm{HCO}^{+}$, and $\mathrm{CS}$ in the local, single-nucleus, ultraluminous infrared galaxy IRAS 13120-5453. We find centrally enhanced $\mathrm{HCN}$ (4-3) emission, relative to $\mathrm{HCO}^{+}$(4-3), but do not find evidence for radiative pumping of HCN. Considering the size of the starburst $(0.5 \mathrm{kpc})$ and the estimated supernovae rate of $\sim 1.2 \mathrm{yr}^{-1}$, the high $\mathrm{HCN} / \mathrm{HCO}^{+}$ratio can be explained by an enhanced $\mathrm{HCN}$ abundance as a result of mechanical heating by the supernovae, though the active galactic nucleus and winds may also contribute additional mechanical heating. The starburst size implies a high $\Sigma_{\mathrm{IR}}$ of $4.7 \times 10^{12} L_{\odot} \mathrm{kpc}^{-2}$, slightly below predictions of radiation-pressure limited starbursts. The HCN line profile has low-level wings, which we tentatively interpret as evidence for outflowing dense molecular gas. However, the dense molecular outflow seen in the HCN line wings is unlikely to escape the Galaxy and is destined to return to the nucleus and fuel future star formation. We also present modeling of Herschel observations of the $\mathrm{H}_{2} \mathrm{O}$ lines and find a nuclear dust temperature of $40 \mathrm{~K}$. IRAS 13120-5453 has a lower dust temperature and $\Sigma_{\mathrm{IR}}$ than is inferred for the systems termed "compact obscured nuclei (CONs)" (such as Arp 220 and Mrk 231). If IRAS 13120-5453 has undergone a CON phase, we are likely witnessing it at a time when the feedback has already inflated the nuclear ISM and diluted star formation in the starburst/active galactic nucleus core.
\end{abstract}

Key words: galaxies: active - galaxies: individual (IRAS 13120-5453) - galaxies: interactions - galaxies: ISM galaxies: starburst

\section{Overview of IRAS 13120-5453 and Dense Gas Tracers}

Star formation rates on $\sim 1 \mathrm{kpc}$ scales are well correlated with the local (molecular) gas surface density (Bigiel et al. 2008), consistent with a scenario in which the stars form out of molecular gas. As cold $\mathrm{H}_{2}$ does not have strong emission, tracer molecules such as $\mathrm{CO}, \mathrm{HCN}$, and $\mathrm{HCO}^{+}$are used to quantify the properties of the molecular ISM. Molecular outflows have been identified in many starbursts and active galactic nuclei (AGNs; e.g., Feruglio et al. 2010; Alatalo et al. 2011; Sturm et al. 2011; Aalto et al. 2012a, 2012b; Feruglio et al. 2013; Veilleux et al. 2013; Cicone et al. 2014; Sakamoto et al. 2014; García-Burillo et al. 2015) and may represent the clearing of the fuel for star formation.

The present study is focused on the properties of the highdensity tracers $\mathrm{HCN}$ and $\mathrm{HCO}^{+}, \mathrm{H}_{2} \mathrm{O}$ emission, and the excitation and kinematics of those tracers in the ultraluminous infrared galaxy (ULIRG), IRAS 13120-5453. Below we discuss these tracers and the general properties of this ULIRG.

\section{1. $\mathrm{HCN}, \mathrm{HCO}^{+}$, and the Star-forming Molecular Gas}

$\mathrm{CO}(1-0)$ is widely used as a tracer of the total molecular gas mass within a galaxy (e.g., Bolatto et al. 2013b); its relatively low critical density $\left(n_{\text {crit }} \approx 10^{2} \mathrm{~cm}^{-3}\right)$ and energy level $(E /$ $\left.k_{\mathrm{B}}=5.5 \mathrm{~K}\right)$ mean it is associated with even the low density molecular gas which is not directly involved in ongoing star formation. Empirically, this has been seen in studies which show the CO luminosity has a nonlinear relation with the star formation rate (as traced by $L_{\mathrm{IR}}[8-1000 \mu \mathrm{m}]$; Gao \& Solomon 2004a). In contrast, the $\mathrm{HCN}(1-0)$ and $\mathrm{HCO}^{+}(1-0)$ emission are linearly correlated with the SFR (e.g., Solomon et al. 1992; Gao \& Solomon 2004a). This, plus the comparatively higher critical densities of the $1 \rightarrow 0$ lines $\left(n_{\text {crit }} \approx 10^{6}\right.$ and $10^{5} \mathrm{~cm}^{-3}$ at $30 \mathrm{~K}$, respectively) suggests the $\mathrm{HCN}$ and $\mathrm{HCO}^{+}$emission trace the dense gas that is actively associated with ongoing star formation.

The excitation of $\mathrm{HCN}$ and $\mathrm{HCO}^{+}$is uncertain in extreme star-forming galaxies. The excitation seems to systematically vary with gas density and the incident UV radiation field (Meijerink et al. 2007), and may vary with the infrared radiation field due to radiative pumping (Aalto et al. 1995). Additionally, the relative $\mathrm{HCN} / \mathrm{HCO}^{+}$abundance can be affected by chemistry driven by X-rays (e.g., Lepp \& Dalgarno 1996) and mechanical heating (Loenen et al. 2008; Kazandjian et al. 2012). Understanding the excitation and abundance of these high $n_{\text {crit }}$ tracers is crucial to accurately 
characterizing the dense molecular gas in star-forming systems (i.e., determining the dense gas fraction and the physical conditions of the dense gas).

Studies of AGN hosts have found evidence for enhanced $\mathrm{HCN}$ emission (relative to $\mathrm{HCO}^{+}$) in both galaxy-integrated and resolved observations (e.g., Kohno et al. 2001; Imanishi et al. 2006, 2007; Davies et al. 2012), which has been interpreted as evidence for the influence of X-ray dominated regions (XDRs) or mechanical heating (Izumi et al. 2016). More recent studies of galaxy-integrated emission have uncovered enhanced $\mathrm{HCN}$ emission in pure starburst and composite systems (Costagliola et al. 2011; Privon et al. 2015), but existing data were not sufficient to suggest a single preferred physical process for the enhancement. Other studies have found evidence for nonlinear relationships of HCN (1-0) with $L_{\mathrm{FIR}}[40-400 \mu \mathrm{m}]$ (García-Burillo et al. 2012), in contrast to the Gao \& Solomon (2004a) picture.

Recent interferometric observations of the (3-2) and (4-3) lines in systems with enhanced HCN emission have uncovered convincing evidence of infrared pumping, via the detection of $v_{2}=1 f$ lines of HCN (e.g., Sakamoto et al. 2010; Costagliola et al. 2013; Imanishi \& Nakanishi 2013; Aalto et al. 2015a, $2015 \mathrm{~b}$ ). The $v_{2}=1 f \mathrm{HCN}(4-3)$ line has a level energy of $1050 \mathrm{~K}$, and is thus unlikely to be collisionally excited. Instead, it has been proposed that mid-infrared pumping, via absorption of $14 \mu \mathrm{m}$ photons, excites this ro-vibrational branch (Ziurys \& Turner 1986; Aalto et al. 1995). It is possible that the radiative pumping may enhance the $v_{2}=0$ emission of lower- $J$ transitions (e.g., Carroll \& Goldsmith 1981), potentially explaining elevated $\mathrm{HCN} / \mathrm{HCO}^{+}$ratios, but this has not yet been confirmed observationally.

Most of the extragalactic $\mathrm{HCN} v_{2}=1 f$ detections are in systems which appear to contain dense, high column, hot $\left(T_{\text {dust }}>100 \mathrm{~K}\right)$ cores (Aalto et al. 2015a). These systems, dubbed "Compact Obscured Nuclei" (CONs), feature compact starbursts and perhaps also deeply buried (Compton-thick) AGN and appear to be optically thick in the mid-infrared. As a result, submillimeter lines may be the only way to probe the inner structure.

Individual galactic star-forming regions have also been found to have elevated $\mathrm{HCN} / \mathrm{HCO}^{+}$, particularly in the circumnuclear disk (CND) of the Galactic center where $\mathrm{HCN} / \mathrm{HCO}^{+} \sim 1.5-2$ (Mills et al. 2013). Despite the presence of $v_{2}=1 f$ emission in the Galaxy's CND, enhanced HCN emission appears to not be driven by IR pumping or XDRs. Mills et al. (2013) state photodissociation region (PDR) models are consistent with the observed ratio, but would likely fail to explain the high gas temperatures seen in the CND (RequenaTorres et al. 2012), while mechanical heating can simultaneously explain both the $\mathrm{HCN} / \mathrm{HCO}^{+}$ratio and the gas temperature. Furthermore, mechanical heating from a jet or outflow has been invoked as the driver of high $\mathrm{HCN} / \mathrm{HCO}^{+}$ ratios in AGN hosts (e.g., Izumi et al. 2013; García-Burillo et al. 2014; Izumi et al. 2015, 2016).

These observational results point to a complex interplay of excitation effects and chemistry-driven abundance variations. The shocks and turbulence resulting from supernovae, as well as AGN- and starburst-driven winds, which can penetrate deep into molecular clouds, may result in elevated $\mathrm{HCN} / \mathrm{HCO}^{+}$ abundance ratios and higher $\mathrm{HCN} / \mathrm{HCO}^{+}$luminosity ratios. Substantial variations in the relative abundances of these tracer molecules and their excitation would bias estimates of the dense gas mass from $\mathrm{HCN}$ luminosities. Atacama Large Millimeter/submillimeter Array (ALMA) observations are needed to resolve the emission from these molecular tracers and link luminosity variations to the underlying nuclear star formation and AGN activity.

\subsection{Water Emission}

Emission from $\mathrm{H}_{2} \mathrm{O}$ molecules appears to be common in extreme star-forming galaxies (Fischer et al. 1999; GonzálezAlfonso et al. 2004, 2008, 2012; Fischer et al. 2010). The submillimeter $\mathrm{H}_{2} \mathrm{O}$ lines appear to require pumping from the far-infrared continuum (González-Alfonso et al. 2014), making water emission a good probe of warm, dusty regions and the far-infrared radiation field in those regions. Based on modeling of the $\mathrm{H}_{2} \mathrm{O}$ lines and agreement with results from millimeter HCN observations, González-Alfonso et al. (2014) argue the water emission is co-spatial with $\mathrm{HCN}$ emission. Additionally they find broad characteristics of the $\mathrm{H}_{2} \mathrm{O}$ emission at submillimeter wavelengths in warm, star-forming galaxies can be explained with dust temperatures $T_{\text {dust }}=55-75 \mathrm{~K}$, a $100 \mu \mathrm{m}$ optical depth $\tau_{100} \sim 0.1$, and a column density of $N_{\mathrm{H}_{2} \mathrm{O}} \sim(0.2-2) \times 10^{17} \mathrm{~cm}^{-2}$, when the highest-lying submillimeter lines (at $>400 \mathrm{~K}$ ) are not detected.

The coupling of $\mathrm{H}_{2} \mathrm{O}$ emission to the infrared radiation field and the co-spatial nature with the $\mathrm{HCN}$ suggests that modeling of the water emission can constrain the dust temperature in the dense molecular gas independently of the infrared SED. This provides vital constraints on the physical conditions in the molecular regions traced by $\mathrm{HCN}$, aiding in the interpretation of the HCN emission, both for systems that are optically thin and optically thick at $100 \mu \mathrm{m}$ (González-Alfonso et al. 2014).

\subsection{Target: IRAS 13120-5453}

IRAS $13120-5453$ is a ULIRG with $L_{\mathrm{IR}}[8-1000 \mu \mathrm{m}]=$ $2.1 \times 10^{12} L_{\odot}$ (Armus et al. 2009) at a distance $D_{L}=144 \mathrm{Mpc}$ $\left(z=0.03112\right.$; angular scale: $\left.0.656 \mathrm{kpc} \operatorname{arcsec}^{-1}\right)$. Several multiwavelength studies have morphologically classified this system as a post-merger, single-nucleus system (Figure 1; Haan et al. 2011; Stierwalt et al. 2013). Kim et al. (2013) applied GALFIT modeling to HST images of the system and found a significant portion of the flux $(\sim 35 \%)$ is in non-axisymmetric structures, consistent with a scenario in which the system has not fully relaxed. A visual inspection of the large-scale morphology shows a faint tidal tail stretching to the north, with multiple loops surrounding the main body of the Galaxy, suggesting the extended regions of the system are re-accreting material from the tidal tails.

The system is optically classified as a Seyfert 2 (Véron-Cetty \& Véron 2001), and the equivalent width of the $6.2 \mu \mathrm{m}$ polycyclic aromatic hydrocarbon $(=0.45 \mu \mathrm{m}$; Stierwalt et al. 2013) suggests the infrared luminosity arises due to a mix of reprocessed radiation from both a starburst and AGN. X-ray observations of IRAS 13120-5453 also find evidence for an AGN, with an estimated $\sim 18 \%$ contribution of the AGN to $L_{\mathrm{IR}}$ (Iwasawa et al. 2011). Nuclear Spectroscopic Telescope Array (NuSTAR) hard X-ray observations of the system are consistent with the presence of a Compton-thick AGN $\left(N_{\mathrm{H}}=3.15_{-1.29}^{+2.23} \times 10^{24} \mathrm{~cm}^{-2}\right.$; Teng et al. 2015) with $L_{\mathrm{AGN}, 2-10 \mathrm{keV}}=1.25 \times 10^{43} \mathrm{erg} \mathrm{s}^{-1}$ and a star formation rate of $\sim 170 M_{\odot} \mathrm{yr}^{-1}$ (the latter determined from the thermal emission and the emission associated with high-mass $\mathrm{X}$-ray binaries). The high obscuration toward the X-ray emitting 


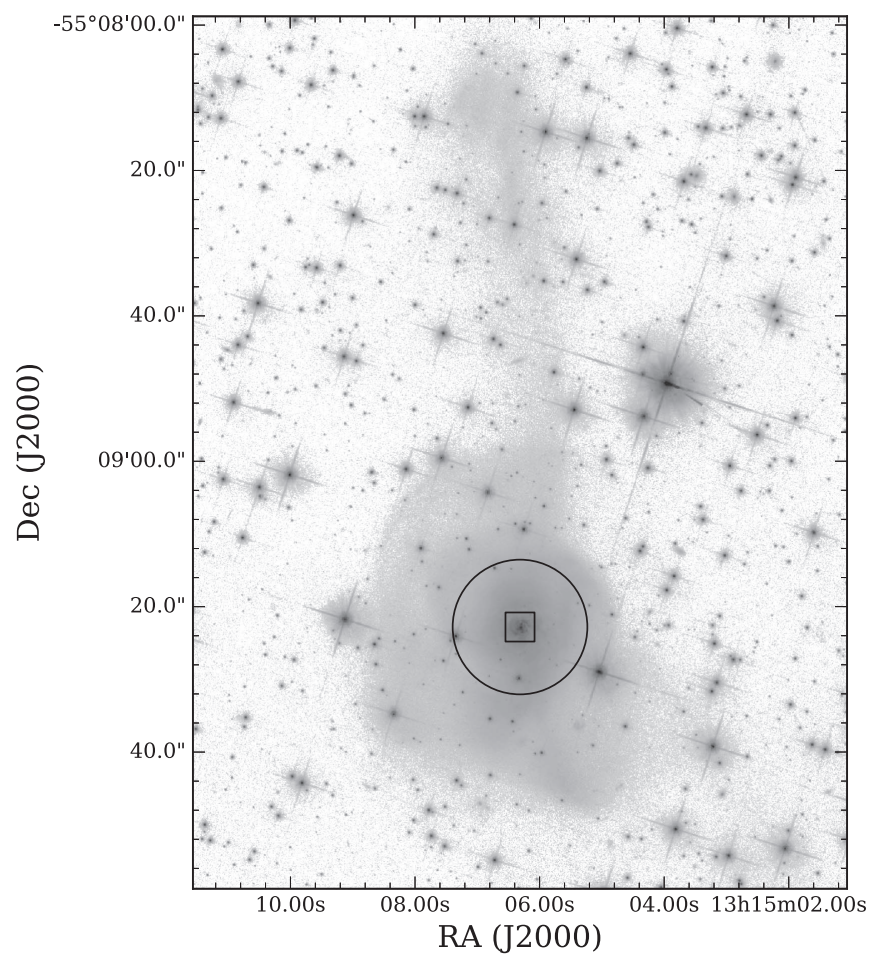

Figure 1. HST/ACS F814W image of IRAS 13120-5453 (A. S. Evans et al. 2016, in preparation), showing the single-nucleus, long tidal tail stretching to the north, and the loops from re-accreted tidal material. The black circle denotes the ALMA Band 7 primary beam. The detected line and continuum emission is concentrated on the nucleus and confined within the black square shown, which denotes the coverage of the panels in Figures 3 and 9.

region is consistent with the optical classification, where we only see the narrow lines. The optical depth of the $9.7 \mu \mathrm{m}$ silicate absorption feature is $\tau_{9.7}=2.52$ (Stierwalt et al. 2014), corresponding to $A_{V} \approx 23$ following the relationship found by Roche \& Aitken (1985) for $\tau_{9.7}$ and $A_{V}$ for the galactic center. We note the obscuration giving rise to the silicate absorption likely occurs outside of the nucleus but within the host galaxy (e.g., GonzálezMartín et al. 2013; Roche et al. 2015) or from absorption within the starburst (Díaz-Santos et al. 2013). The [C II] emission is suppressed relative to the far-infrared ([C II] $] / L_{\mathrm{FIR}}=$ $(6.3 \pm 0.1) \times 10^{-4}$; Díaz-Santos et al. 2013). Spitzer observations were used to place an upper limit on the mid-infrared size of the starburst of $2.68 \mathrm{kpc}$ (Díaz-Santos et al. 2010), leading to a lower limit on the infrared luminosity surface density of $3.0 \times 10^{11} L_{\odot} \mathrm{pc}^{-2}$. Using the upper limit on the size, IRAS 13120-5453 lies below the compact starburst/[C II] suppression model of Díaz-Santos et al. (2013), though this may be due to the underestimation of the IR luminosity surface density.

In this paper we present new ALMA Band 7 observations of the $v_{2}=0,1 \mathrm{HCN}(4-3), v_{2}=0 \mathrm{HCO}^{+}(4-3)$ and $\mathrm{CS}(7-6)$ lines (Section 2), then discuss the excitation of these dense gas tracers and a tentatively detected outflow (Section 3). Next we show results from modeling of the $\mathrm{H}_{2} \mathrm{O}$ lines observed with Herschel (Section 4), aimed at constraining the dust temperature of the dense ISM. We then explore the nuclear kinematics of the system (Section 5), and the ISM properties and starburst size inferred from our ALMA detection of the $\sim 333 \mathrm{GHz}$ continuum emission (Section 6). We conclude by discussing the implications of the ISM properties for the fate of the starburst in IRAS 13120-5453 (Section 7). Where appropriate, values were computed assuming a WMAP-5 cosmology
$\left(H_{0}=70 \mathrm{~km} \mathrm{~s}^{-1} \mathrm{Mpc}^{-1}, \quad \Omega_{\text {vacuum }}=0.72, \Omega_{\text {matter }}=0.28\right.$; Hinshaw et al. 2009), with corrections for the three-attractor model of Mould et al. (2000).

\section{Observations}

\subsection{Atacama Large Millimeter/submillimeter Array}

ALMA observations were carried out on 2014 May 18 in the C32-5 configuration, as part of project \#2012.1.00817.S (PI: Aalto) with an on-source time of 19.1 minutes. These data have projected baseline lengths between 23 and $625 \mathrm{~m}$. The observing setup consisted of four independent spectral windows: one each tuned to the redshifted frequencies of $\mathrm{HCN}(4-3), \mathrm{HCO}^{+}$(4-3) and two centered at frequencies of 331.9 GHz (covering CS (7-6)) and 333.7 GHz (Figure 2). All four spectral windows had bandwidths of $1.875 \mathrm{GHz}$. The weather conditions were good, with a precipitable amount of water vapor of $0.8 \mathrm{~mm}$. The median on-source system temperature was $180 \mathrm{~K}$. Observations were calibrated and imaged in a standard fashion using the Common Astronomy Software Applications (McMullin et al. 2007). The bandpass response of the array was calibrated using the quasar J10372934. The flux calibration was set by observations of Ganymede, using the Butler-JPL-Horizons 2012 model, as described in ALMA Memo 594. ${ }^{14}$ The absolute flux calibration is expected to be better than $10 \%$. The gain calibration was done with the quasar J1329-5608. An iteration of phase selfcalibration was possible since the continuum of IRAS 131205453 is strong enough to allow us to derive gain solutions on a time interval of $20 \mathrm{~s}$. The phase center is $13^{\mathrm{h}} 15^{\mathrm{m}} 06^{\mathrm{s}} .316$ $-55^{\mathrm{d}} 09^{\mathrm{m}} 22^{\mathrm{s}} .79$ (J2000). The data were imaged using Briggs weighting (robust $=0.5$; Briggs 1995) and the resulting resolution of the data cubes was 0 ". $50 \times 0$ "! $28(\sim 325 \times 180$ pc) at a position angle of $-75^{\circ}$ with an rms sensitivity of $1.2 \mathrm{mJy} \mathrm{beam}^{-1}$ at $20 \mathrm{~km} \mathrm{~s}^{-1}$ spectral resolution. Continuumfree cubes were created by subtracting a linear baseline fit to the line-free channels in the image plane (CASA task imcontsub). We adopt a rest frequency of $354.526 \mathrm{GHz}$ for $\mathrm{HCN}(4-3), \quad 356.754 \mathrm{GHz}$ for $\mathrm{HCO}^{+}(4-3)$, and $342.883 \mathrm{GHz}$ for CS (7-6). The $v_{2}=1 \mathrm{HCN}$ (4-3) doublet, included in the two higher frequency spectral windows, have rest frequencies of $354.460 \mathrm{GHz}\left(v_{2}=1 e\right)$ and $356.256 \mathrm{GHz}$ $\left(v_{2}=1 f\right)$; the former component is underneath the $v_{2}=0$ line, while the latter is $420 \mathrm{~km} \mathrm{~s}^{-1}$ from the $\mathrm{HCO}^{+}$(4-3) line (see Figure 2). When discussing the vibrational lines, we refer to them as HCN vibrational or explicitly denote the line as $v_{2}=1 f$. The $v=0$ rotational lines will be referred to with their J-level transitions. All rest frequencies were obtained from the JPL Submillimeter, Millimeter, and Microwave Spectral Line Catalog (Pickett et al. 1998) through Splatalogue.

\subsection{Herschel Space Observatory}

IRAS 13120-5453 was observed using the Photodetector Array Camera and Spectrometer (PACS; Poglitsch et al. 2010) and the Spectral and Photometric Imaging Receiver (SPIRE; Griffin et al. 2010) on the Herschel Space Observatory. The PACS observations were performed in high spectral sampling range spectroscopy mode on 2012 July 19 as part of the

\footnotetext{
14 https://science.nrao.edu/facilities/alma/aboutALMA/Technology/ ALMA_Memo_Series/alma594/abs594
} 


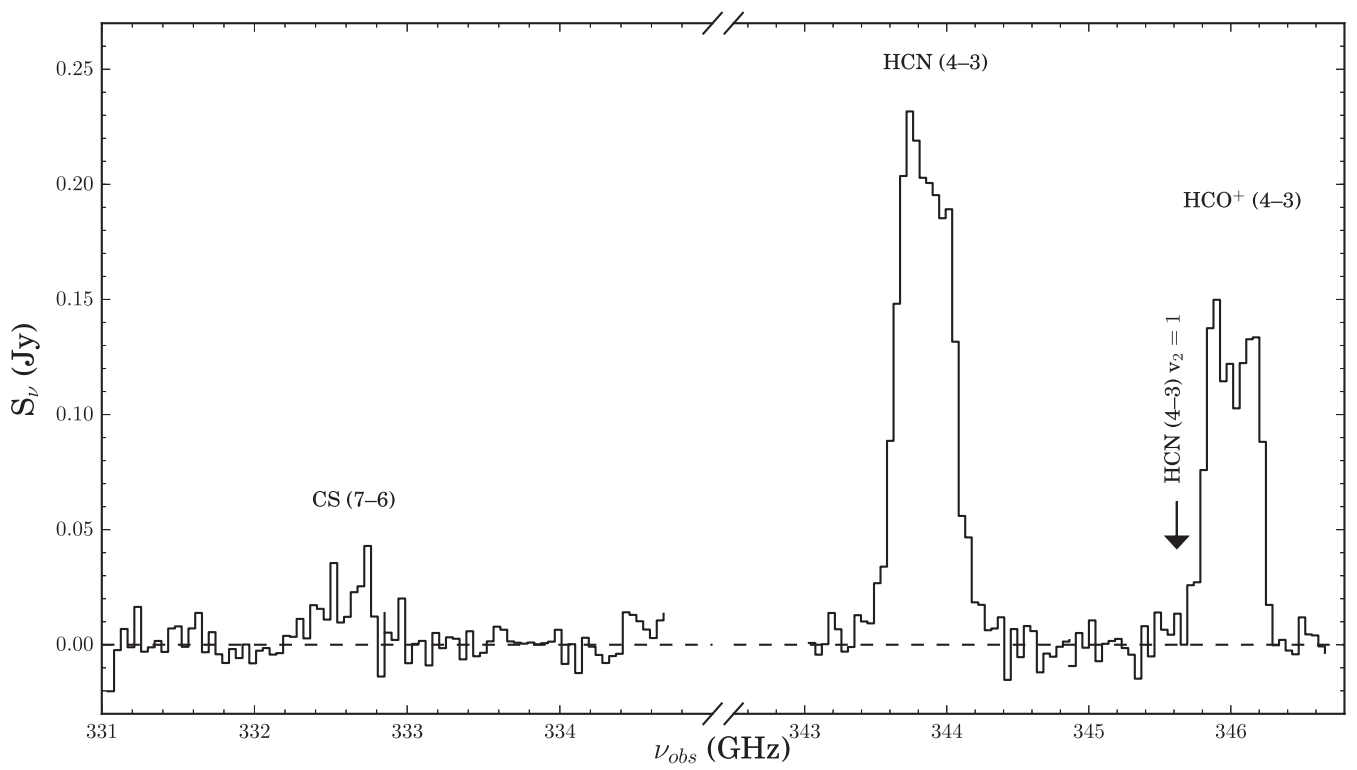

Figure 2. Integrated spectra from the ALMA observations, continuum-subtracted and measured from a 3 arcsec diameter circular region centered on the nucleus. The locations of detected and expected lines are marked; their measured properties are given in Table 1 . The arrow and label marks the location of the HCN $(4-3) v_{2}=1 f$ line; emission is seen at those frequencies, but we attribute it to $\mathrm{HCO}^{+}$(4-3) emission associated with a molecular outflow (Section 3.2.1).

Hermolirg OT2 project (PI: E. González-Alfonso; ObsIDs: 1342248346 \& 1342248347$)$.

The SPIRE observation was performed on 2011 January 05 as part of the OT key program Hercules (PI: P.P. van der Werf; ObsID: 1342212342) with a single pointing centered on IRAS 13120-5453. The observation was conducted in high spectral resolution, sparse image sampling mode with a resolution of $1.2 \mathrm{GHz}$ in both observing bands $(447-989 \mathrm{GHz}$ and 958-1545 GHz). A total of 29 repetitions (58 FTS scans) were performed, resulting in a total on-source integration time of 3863 s.

The data reduction was done with the Herschel interactive processing environment (HIPE; Ott 2010) version 14.0.1. The PACS observations were reduced using the standard telescope normalization pipeline for chopped line scans and short range scans. Each spaxel in the PACS $5 \times 5$ spaxel array is a square with 9!.4 to each side. At a distance of $144 \mathrm{Mpc}$ this corresponds to $\sim 6 \mathrm{kpc}$, making the nuclear far-IR emission in IRAS 13120-5453 spatially unresolved in the central spaxel. As the point-spread function of the spectrometer is larger than the central spaxel, the central spectrum was extracted using the point source correction task available in HIPE 14.0.1. To compensate for small pointing offsets and jitter that might move flux out of the central spaxel, this extracted spectrum was scaled to the integrated flux level of the central $3 \times 3$ spaxels. The data reduction for the SPIRE observation was done with the standard single pointing pipeline.

To extract the line fluxes of the SPIRE observation a bootstrap method was used. A total of 58 scans were drawn randomly, with replacement, from the original observation and then averaged together. For each detector, a polynomial baseline was then subtracted from the spectrum before simultaneously fitting the spectral lines using Gaussian profiles convolved with the instrumental response (a sinc function). This procedure was repeated 1000 times and a Gaussian was fitted to the resulting flux distribution of each line to obtain its mean line flux and standard deviation.

\section{The Dense Gas Tracers}

Emission from $\mathrm{HCN}(4-3), \mathrm{HCO}^{+}$(4-3) and $\mathrm{CS}$ (7-6) were detected at 215, 243, and 20 $\sigma$, respectively (Figure 2). The emission from these species is compact $(1 / ! 8,1.2 \mathrm{kpc})$ and centrally concentrated (Figure 3 ). We did not see clear evidence for strong emission of the $v_{2}=1 f \mathrm{HCN}$ (4-3) vibrational transition, but see Section 3.2.1 for discussion. We also detect the $333 \mathrm{GHz}$ continuum emission at $225 \sigma$ (Table 1). Measured parameters are provided in Table 1. Properties of IRAS 13120-5453 derived from these observations are given in Table 2 and discussed in detail in later sections.

\subsection{Comparison with Single-dish Measurements}

IRAS 13120-5453 was observed by Zhang et al. (2014) with the APEX $12 \mathrm{~m}$ telescope. Using a conversion of $41 \mathrm{Jy} \mathrm{K}^{-115}$, their $\mathrm{HCN}(4-3)$ and $\mathrm{HCO}^{+}$(4-3) fluxes are $82 \pm 12 \mathrm{Jy} \mathrm{km} \mathrm{s}^{-1}$ and $66 \pm 12 \mathrm{Jy} \mathrm{km} \mathrm{s}^{-1}$, respectively. Our HCN flux agrees with theirs, suggesting we are recovering the total flux with these ALMA data. Our $\mathrm{HCO}^{+}$flux is $\sim 25 \%$ lower than theirs, indicating we may be resolving out some extended flux on scales $\gtrsim 8^{\prime \prime}$ (the largest recoverable scale for this ALMA configuration and observing frequency) and $\lesssim 18^{\prime \prime}$ (the beam size of APEX at these frequencies), though emission on scales $\mathrm{4}^{\prime \prime}$ will also be affected by filtering. The possible effects of this are further discussed in Section 3.2.2.

Zhang et al. (2014) quote an upper limit of $<36.6 \mathrm{Jy} \mathrm{km} \mathrm{s}^{-1}$ for the CS (7-6) line; our measured line flux is nearly a factor of 10 below their upper limit, and thus consistent.

Using our detections of several dense gas tracers we investigate the excitation of $\mathrm{HCN}$ and $\mathrm{HCO}^{+}$, as well as the spatial variations of the $\mathrm{HCN} / \mathrm{HCO}^{+}$ratio (Section 3.2). These ALMA data further reveal tentative evidence for outflowing dense molecular gas, through wings on the $\mathrm{HCN}$ and $\mathrm{HCO}^{+}$ lines (Section 3.3).

\footnotetext{
15 Obtained from the APEX website: http://www.apex-telescope.org/ telescope/efficiency/.
} 

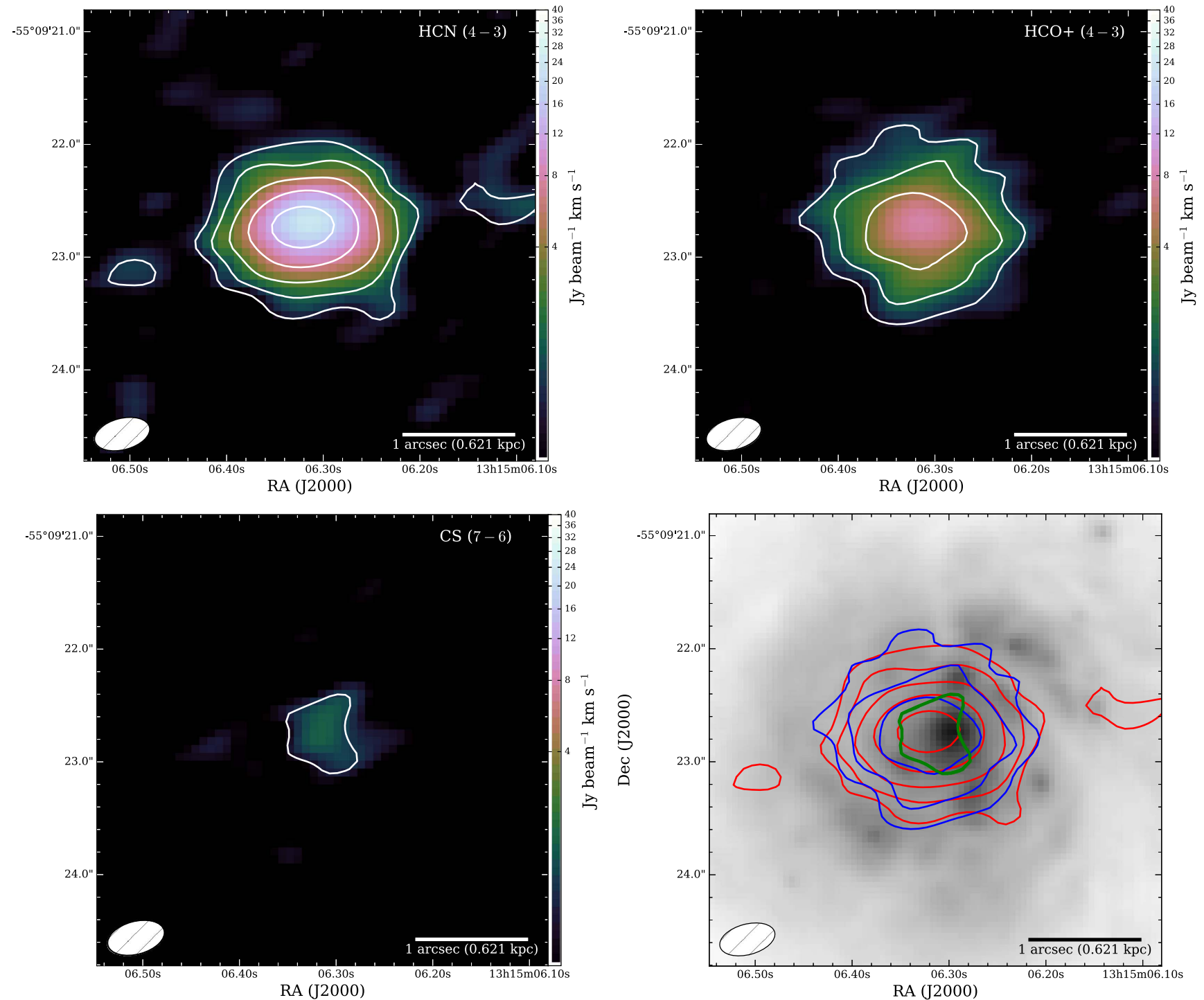

Figure 3. Upper left: $\mathrm{HCN}(4-3)$ total intensity map. Upper right: $\mathrm{HCO}^{+}(4-3)$ total intensity map. Lower left: CS (7-6) total intensity map. Lower right: $H S T$ /ACS F814W image of the central $\sim 7 \mathrm{kpc}$ of IRAS $13120-5453$, with the total intensity of $\mathrm{HCN}(4-3), \mathrm{HCO}^{+}$(4-3), and CS (7-6) shown in red, blue, and green contours, respectively, to illustrate their relationship to each other and the underlying optical continuum emission. The three color scale figures all utilize the same brightness scaling to illustrate the relative intensity of the three molecular lines. In all figures, the size of the ALMA synthesized beam is shown in the lower-left. The emission from these tracers is confined to a molecular disk with an overall extent of $\sim 1.2 \mathrm{kpc}$. The contour levels in all four panels begin at $1 \mathrm{Jy}^{\mathrm{beam}}{ }^{-1} \mathrm{~km} \mathrm{~s} \mathrm{~s}^{-1}$ and increase by factors of 2. The relative astrometry of the HST/ACS image is uncertain to roughly $1^{\prime \prime}$, so the peak of the molecular emission may be consistent with the position of the optical nucleus.

Table 1

Measured Molecular Line and Continuum Properties

\begin{tabular}{lcc}
\hline \hline & Integrated Flux & $\begin{array}{c}\mathrm{FWHM}^{\mathrm{a}} \\
\left(\mathrm{km} \mathrm{s}^{-1}\right)\end{array}$ \\
\hline $\mathrm{HCN}(4-3)$ & $86.2 \pm 0.4 \mathrm{Jy} \mathrm{km} \mathrm{s}^{-1}$ & 380 \\
$\mathrm{HCN}(4-3) v_{2}=1 f$ & $<0.27^{\mathrm{b}} \mathrm{Jy} \mathrm{km} \mathrm{s}^{-1}$ & $\ldots$ \\
$\mathrm{HCO}^{+}(4-3)$ & $48.6 \pm 0.2 \mathrm{Jy} \mathrm{km} \mathrm{s}^{-1}$ & 360 \\
$\mathrm{CS}(7-6)$ & $4.2 \pm 0.2 \mathrm{Jy} \mathrm{km} \mathrm{s}^{-1}$ & 250 \\
\hline $333 \mathrm{GHz}$ & $89.8 \pm 0.4 \mathrm{mJy}$ & $\ldots$
\end{tabular}

Notes. Col 1-Line identification or continuum frequency, Col 2-Integrated flux (for lines) or flux density (for continuum), $\mathrm{Col} 3$-Measured full-width of the emission line at half of the observed peak value.

${ }^{a}$ Width measured directly from the line profiles.

b $1 \sigma$ upper limit, assuming a boxcar line with a width of $200 \mathrm{~km} \mathrm{~s}^{-1}$, motivated by the width of detected $v_{2}=1 f$ lines in other systems (Aalto et al. 2015a).

\subsection{Excitation of $\mathrm{HCN}$ and $\mathrm{HCO}^{+}$}

In Figure 4 we show the spatially resolved $L_{\mathrm{HCN}(4-3)}^{\prime} /$ $L_{\mathrm{HCO}^{+}(4-3)}^{\prime}$ ratio and its $\mathrm{S} / \mathrm{N}$. The ratio map was created by dividing the total intensity map of $\mathrm{HCN}(4-3)$ by the total intensity map of $\mathrm{HCO}^{+}(4-3)$, masking out the regions where $\mathrm{HCO}^{+}(4-3)$ was not detected at $\geqslant 3 \sigma$. We find that the $\mathrm{HCN} /$ $\mathrm{HCO}^{+}$ratio peaks at $\sim 2.8$ over the nucleus (in the central resolution element: $325 \times 180 \mathrm{pc}$ ), and decreases to $\sim 1$ off the nucleus. We measure a spatial- and velocity-integrated $\mathrm{HCN}(4-3) / \mathrm{HCO}^{+}(4-3)$ ratio of $1.77 \pm 0.01$.

How do these ratios compare with the ratios expected for starburst galaxies? If we cross-correlate the single-dish $\mathrm{HCN}(4-3)$ and $\mathrm{HCO}^{+}$(4-3) measurements of Zhang et al. (2014) with the $6.2 \mu \mathrm{m}$ PAH EQW measurements of Stierwalt et al. (2013) and take the PAH EQW as a proxy for midinfrared AGN dominance $(\mathrm{EQW}<0.2$ are dominated by 
Table 2

Derived Nuclear Properties

\begin{tabular}{lrl}
\hline \hline Quantity & Value & Units \\
\hline$M_{\text {dyn }}[\mathrm{HCN}(4-3), R<0.5 \mathrm{kpc}]$ & $1.2(\sin i)^{-2} \times 10^{10}$ & $M_{\odot}$ \\
$M_{\text {dyn }}\left[\mathrm{HCO}^{+}(4-3), R<0.5 \mathrm{kpc}\right]$ & $1.0(\sin i)^{-2} \times 10^{10}$ & $M_{\odot}$ \\
$\Sigma_{\text {dyn }}[R<0.5 \mathrm{kpc}]^{\mathrm{a}}$ & $1.2(\sin i)^{-2} \times 10^{10}$ & $M_{\odot} \mathrm{kpc}^{-2}$ \\
$M_{\text {ISM }}[\text { total, } 333 \mathrm{GHz}]^{\mathrm{b}}$ & $(3.3 \pm 0.7) \times 10^{10}$ & $M_{\odot}$ \\
$M_{\text {ISM }}[\text { total, 333 GHz}]^{\mathrm{c}}$ & $3 \times 10^{10}$ & $M_{\odot}$ \\
$M_{\text {ISM }}[R<0.5 \mathrm{kpc}, 333 \mathrm{GHz}]^{\mathrm{b}}$ & $(1.4 \pm 0.4) \times 10^{10}$ & $M_{\odot}$ \\
$M_{\text {ISM }}\left[R<0.5 \mathrm{kpc}, \mathrm{H}_{2} \mathrm{O} \text { modeling }\right]^{\mathrm{d}}$ & $7.5 \times 10^{9}$ & $M_{\odot}$ \\
$\Sigma_{\text {ISM }, 50}[$ within half-light radius of & $5.7 \times 10^{10}$ & $M_{\odot} \mathrm{kpc}^{-2}$ \\
$333 \mathrm{GHz}$ emission] & & \\
$\sum_{\text {IR,50 }}{ }^{\mathrm{e}}[$ within half-light radius of & $4.7 \times 10^{12}$ & $L_{\odot} \mathrm{kpc}^{-2}$ \\
$333 \mathrm{GHz}$ emission] & & \\
\hline
\end{tabular}

Notes.

${ }^{\text {a }}$ Calculated using the mean $M_{\mathrm{dyn}}$ from $\mathrm{HCN}$ and $\mathrm{HCO}^{+}$.

${ }^{b}$ Calculated using the empirical relation from Scoville et al. (2014). This calibration assumes the $\mathrm{H}$ I mass is equal to $50 \%$ of the molecular mass. The Scoville et al. (2016) relation removes the H I mass from the calibration, resulting in a $1 / 3$ reduction in the inferred mass.

${ }^{\mathrm{c}}$ Estimated by computing a dust mass from the $333 \mathrm{GHz}$ continuum emission with the temperature derived from the $\mathrm{H}_{2} \mathrm{O}$ modeling and assuming a gas-todust ratio of 100 .

d Calculated from the $\mathrm{H}_{2}$ column inferred from the modeling of $\mathrm{H}_{2} \mathrm{O}$ lines.

e Calculated by taking the $80 \%$ of $L_{\mathrm{IR}}$ estimated by Díaz-Santos et al. (2010) to originate within the nuclear starburst, and assuming the $L_{\mathrm{IR}}$ follows the distribution of the submillimeter continuum emission.

AGN), the star-forming galaxies have $\mathrm{HCN} / \mathrm{HCO}^{+}$ratios of between 0.2 and 1.5. Thus, the extended emission in IRAS 13120-5453 has a ratio consistent with these starburstdominated systems. The high $\mathrm{HCN} / \mathrm{HCO}^{+}$ratio over the nucleus may point to different excitation conditions and/or $\mathrm{HCN} / \mathrm{HCO}^{+}$abundance ratios, co-spatial with the AGN and nuclear starburst. The line ratios for the nucleus are consistent with what is seen for other AGN hosts (Izumi et al. 2016).

We now discuss the potential mechanisms that could plausibly result in an elevated $\mathrm{HCN} / \mathrm{HCO}^{+}$ratio.

\subsubsection{Limits on Vibrational HCN Emission}

Rotational-vibrational lines of $\mathrm{HCN}\left(v_{2}=1 f, \mathrm{~J}=4 \rightarrow 3\right.$ or $3 \rightarrow 2$ ) have now been detected in eight galaxies (Sakamoto et al. 2010; Imanishi \& Nakanishi 2013; Aalto et al. 2015a, 2015b; S. Aalto et al. 2016, in preparation). The systems have compact nuclei and high implied infrared luminosity surface densities. Based on the high infrared luminosity and low [C II]/ $L_{\text {FIR }}$ ratio (which has been shown to be correlated with starburst luminosity density Díaz-Santos et al. 2013), IRAS 13120-5453 was viewed as a likely candidate for the vibrational HCN lines.

However, we do not detect the $v_{2}=1 f \mathrm{HCN}(4-3)$ line in IRAS 13120-5453, with a $3 \sigma$ upper limit of $0.81 \mathrm{Jy} \mathrm{km} \mathrm{s}^{-1}$, assuming a linewidth of $200 \mathrm{~km} \mathrm{~s}^{-1}$. We find the $v_{2}=0$ / $v_{2}=1 f$ ratio to be $>100$, in contrast to measured ratios of $4-10$ when the $v_{2}=1 f$ line is detected (Aalto et al. 2015a).

The $\mathrm{HCO}^{+}$(4-3) line has a small "shoulder" on the red side (Figure 5); we interpret this as outflowing dense molecular gas (see Section 3.3), but it could plausibly be attributed to the $v_{2}=1 f$ line. If this feature is in fact the $\operatorname{HCN}(4-3) v_{2}=1 f$ line, we find a flux of $2 \mathrm{Jy} \mathrm{km} \mathrm{s}^{-1}$, which is a factor of $\sim 40$ fainter than the main $\mathrm{HCN}$ (4-3) line. However, as is evident from the PV diagram for $\mathrm{HCO}^{+}$(4-3) (Figure 5), the highvelocity emission is not co-spatial with the center of the system, not consistent with expectations for emission from a vib-rotational line, which should be centered on the nucleus (e.g., Aalto et al. 2015a). Thus, we conclude the line wing on $\mathrm{HCO}^{+}(4-3)$ is not the $v_{2}=1 f \mathrm{HCN}$ (4-3) line.

Is the $14 \mu \mathrm{m}$ luminosity surface density high enough to expect appreciable infrared pumping? Using Spitzer IRS spectroscopy, Díaz-Santos et al. (2010) found IRAS 13120-5453 to have an unresolved core with a size of $\leqslant 2.68 \mathrm{kpc}$ (FWHM) at $13.2 \mu \mathrm{m}$. Approximately $80 \%$ of the mid-infrared emission arises in this core. Spitzer observations of IRAS 13120-5453 by Inami et al. (2013) show a $14 \mu \mathrm{m}$ flux of $F_{14}=0.5 \mathrm{Jy}$. If we take the unresolved portion of the $14 \mu \mathrm{m}$ emission and the area we infer from our ALMA observation of the $330 \mathrm{GHz}$ continuum (FWHM of $0.56 \mathrm{kpc} \times 0.49 \mathrm{kpc}$; Section 6), we estimate a $14 \mu \mathrm{m}$ luminosity surface density of $\Sigma_{14} \sim 2.6 \times 10^{11} L_{\odot} \mathrm{kpc}^{-2}$. This is approximately two to three dex below the lower limit of the $14 \mu \mathrm{m}$ surface brightness derived for sources with detected $v_{2}=1 f \mathrm{HCN}$ lines in (Aalto et al. 2015a). Applying an extinction correction to $\Sigma_{14}$ for IRAS 13120-5453 would reduce the discrepancy, but we have no evidence to suggest the mid-infrared emission is being absorbed behind a significant screen of cooler dust. The significantly lower $\Sigma_{14}$ in IRAS 13120-5453 suggests the $14 \mu \mathrm{m}$ continuum may not be effective at radiatively pumping $\mathrm{HCN}$.

Several detections of the $\mathrm{HCN} v_{2}=1 f$ lines occur in systems where the $\mathrm{HCN}$ and $\mathrm{HCO}^{+}$emission is strongly self-absorbed, consistent with a scenario in which the nuclear gas has high density and a high column (Aalto et al. 2015a). There is evidence for some self-absorption in IRAS 13120-5453, but it is not nearly as significant as seen in CONs with detected $v_{2}=1 f$ emission. The integrated line profile (Figure 2) does show a dip in the center, which could be the result of some foreground absorption.

We have performed some exploratory large velocity gradient (LVG) modeling of the $\mathrm{HCN}(4-3), \mathrm{HCO}^{+}$(4-3), and CS (7-6) lines using the Radex and DESPOTIC codes (van der Tak et al. 2007; Krumholz 2014). We ran grids of models covering a range of densities $\left(\log _{10}\left(n / \mathrm{cm}^{-3}\right)=2-7\right)$, column densities $\left(\log _{10}\left(N_{\mathrm{H}_{2}} / \mathrm{cm}^{-2}\right)=21-25\right)$, and relative $\mathrm{HCN} / \mathrm{HCO}^{+}$abundances $\left(10^{-3}-10^{3}\right)$. While the solutions are under-constrained and so we cannot propose "best" values for the system, solutions which matched the observed $\operatorname{HCN}(4-3)$ / $\mathrm{HCO}^{+}$(4-3) value of 2.8 over the nucleus required relative $\mathrm{HCN} / \mathrm{HCO}^{+}$abundances $\gtrsim 10$. This is similar to the result of Izumi et al. (2016), who find $\mathrm{HCN} / \mathrm{HCO}^{+}$abundance ratios of a few to $\gtrsim 10$ are needed to explain the observed $\mathrm{HCN} / \mathrm{HCO}^{+}$ in $\mathrm{AGN}$ hosts, while $\mathrm{HCN} / \mathrm{HCO}^{+}$abundance ratios of $\sim 1$ can explain the emission in starburst galaxies. As we will discuss in Section 7, this abundance enhancement is suggestive of mechanical heating from the nuclear starburst. Measurements of additional transitions of $\mathrm{HCN}$ and $\mathrm{HCO}^{+}$are needed to perform more detailed $\mathrm{LVG}$ modeling to simultaneously constrain the $\mathrm{H}_{2}$ density and relative abundance of each species while also constraining the excitation of these tracer molecules.

\subsubsection{Missing Flux and the $\mathrm{HCN} / \mathrm{HCO}^{+}$Ratio}

What effect does the missing flux in the $\mathrm{HCO}^{+}(4-3)$ line have on our interpretation of line ratios? Zhang et al. (2014) find a $\mathrm{HCN} / \mathrm{HCO}^{+}$ratio of $1.2 \pm 0.3$, somewhat lower than what we find here. If we assume, as a worst-case scenario, that the $17 \mathrm{Jy} \mathrm{km} \mathrm{s}^{-1}$ difference in $\mathrm{HCO}^{+}$(4-3) flux between our 

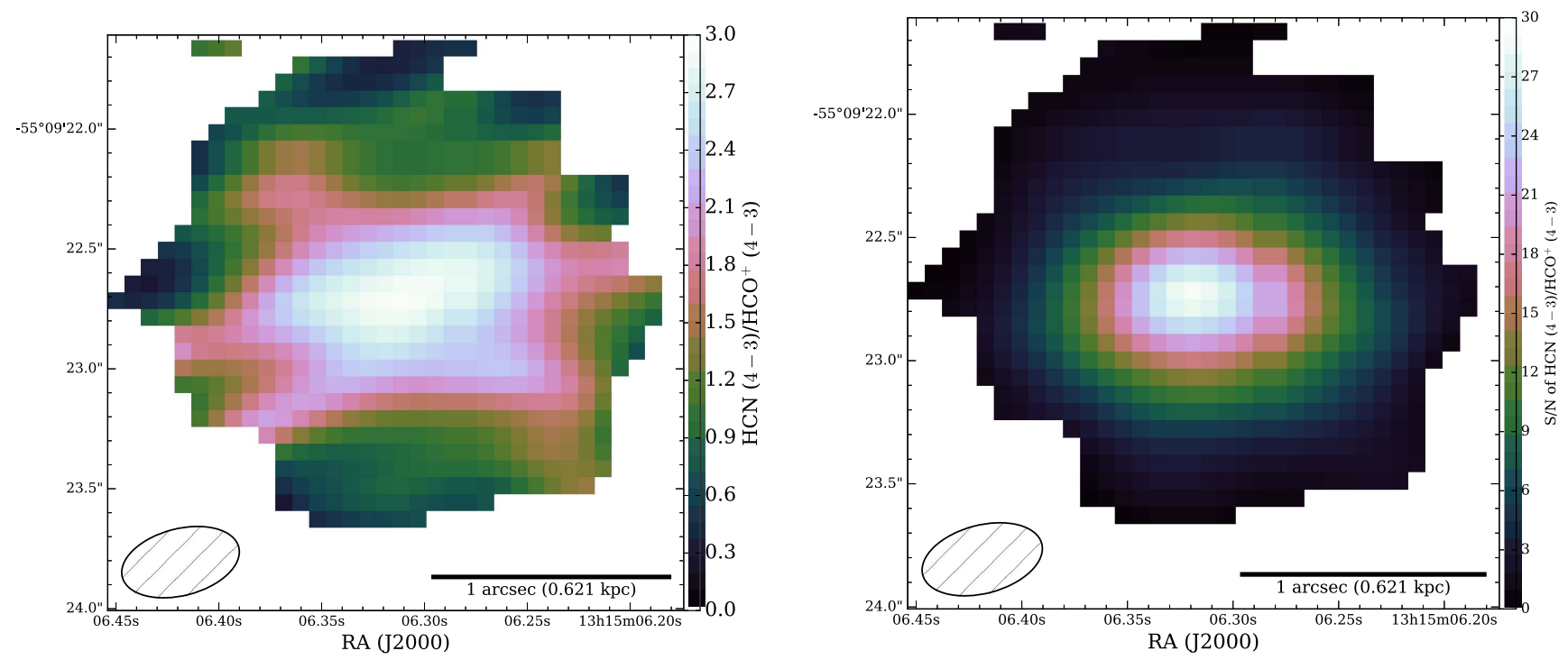

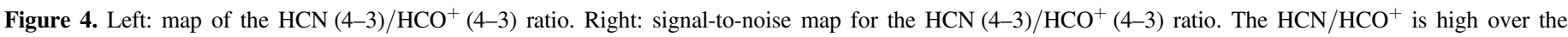
nucleus (central $\sim 300 \mathrm{pc}$ ) and shows a strong (factor of $\sim 3$ ) decrease in the outer portion of the disk.

measurements and those of Zhang et al. (2014) was uniformly resolved out in a $4^{\prime \prime}$ region (the scale on which filtering may start to affect these observations), the contribution of this emission to the central resolution element is $0.15 \mathrm{Jy} \mathrm{km} \mathrm{s}{ }^{-1}$. This contribution would only increase the $\mathrm{HCO}^{+}$(4-3) flux in the central resolution element by $\sim 3 \%$ and so would not substantially affect the ratio at the center of the emission. At larger radii, where the $\mathrm{HCN}$ and $\mathrm{HCO}^{+}$emission is fainter, the potential contribution is more significant, but still amounts to $\lesssim 15 \%$. Thus, we conclude that the missing $\mathrm{HCO}^{+}$(4-3) flux does not significantly influence our spatially resolved determination of the $\mathrm{HCN} / \mathrm{HCO}^{+}$line ratio.

\subsection{Dense Molecular Outflows}

We see wings $\left(\sim 300 \mathrm{~km} \mathrm{~s}^{-1}\right)$ on both the $\mathrm{HCN}(4-3)$ and $\mathrm{HCO}^{+}$(4-3) lines (Figure 5). The $\mathrm{HCN}$ emission appears to have both blue and redshifted wings while, in contrast, $\mathrm{HCO}^{+}$ appears to only have a small amount of emission in the redshifted wing. The $\mathrm{HC}_{3} \mathrm{~N}(39-38)$ line lies at an observed frequency of $\sim 344.1 \mathrm{GHz}$ and can possibly contribute to emission on the blue side of HCN (4-3). However, the imaging of the line wings from the channel shows it is also spatially offset from the nucleus, while we could expect $\mathrm{HC}_{3} \mathrm{~N}$ (39-38) to be centrally concentrated. Thus we conclude that the line wings are not being contaminated by $\mathrm{HC}_{3} \mathrm{~N}(39-38)$.

In Figure 5 we show position-velocity diagrams for both species, taken along the observed disk major axis $\left(\mathrm{PA}=94^{\circ}\right)$. The solid white lines denote the best-fit rotation curve obtained from modeling the data cubes with GalPak ${ }^{3 \mathrm{D}}$ (Bouché et al. 2015). ${ }^{16}$ We also show the expected virial range for the gas (e.g., García-Burillo et al. 2015), defined as a combination of the circular motion, velocity dispersion, and a contribution from in-plane non-circular motion (taken as having a magnitude of $50 \%$ of the circular velocity). The velocities identified as outflows (denoted in Figure 5 by the red lines) lie outside the virial range predicted from the kinematic modeling, indicating they are not participating in quiescent motion within the molecular disk. The details of the kinematic fits to the

\footnotetext{
${ }^{16}$ http://galpak.irap.omp.eu/
}

$\mathrm{HCN}(4-3)$ and $\mathrm{HCO}^{+}$(4-3) lines differ somewhat; this may be due to differential optical depths for the two lines, but they provide consistent estimates for the observed rotation curve and the virial range. Thus, from Figure 5, we conclude the emission isolated as outflows is deviating from the rotation curve seen in the gas.

Based on the velocity channels identified as outflows, approximately $4 \%$ of the detected HCN flux is associated with outflowing gas, while only $1 \%$ of the $\mathrm{HCO}^{+}$flux is in outflows. The luminosity in the outflow is $L_{\mathrm{HCN}(4-3) \text {,outflow }}^{\prime} \approx 2 \times 10^{7} \mathrm{~K} \mathrm{~km} \mathrm{~s}^{-1} \mathrm{pc}^{2}$ and $L_{\mathrm{HCO}^{+}(4-3) \text {,outflow }}^{\prime} \approx 3 \times 10^{6} \mathrm{~K} \mathrm{~km} \mathrm{~s}^{-1} \mathrm{pc}^{2}$. Despite the fact that we are missing $25 \%$ of the single-dish $\mathrm{HCO}^{+}$flux, we are not likely to be missing outflowing gas, given the spatial filtering occurs on scales of of $>4^{\prime \prime}$ and due to the fact that the observed HCN outflow is confined to a region only a few synthesized beams across.

The outflow velocities are modest, spanning $200-400 \mathrm{~km} \mathrm{~s}^{-1}$ in $\mathrm{HCN}$, and $200-300 \mathrm{~km} \mathrm{~s}^{-1}$ in $\mathrm{HCO}^{+}$(Figure 5). With the present data we cannot rule out the presence of dense outflows at the high velocities $\left(\sim 1200 \mathrm{~km} \mathrm{~s}^{-1}\right)$ seen in $\mathrm{OH}$ (Veilleux et al. 2013), though we do not see emission at intermediate velocities. Other $\mathrm{HCO}^{+}$(4-3) observations (K. Sliwa 2016, in preparation) find blueshifted emission at a velocity of $\sim 1100 \mathrm{~km} \mathrm{~s}^{-1}$, but it is unclear if this is $\mathrm{HCO}^{+}$associated with the $\mathrm{OH}$ outflow or emission from another molecular species.

\subsubsection{Velocity-resolved $\mathrm{HCN} / \mathrm{HCO}^{+}$Ratio}

In addition to positional variations, the $\mathrm{HCN} / \mathrm{HCO}^{+}$ratio varies as a function of velocity both for the entire source (Figure 6, middle) and the central resolution element (Figure 6, bottom). In particular, the ratio appears most elevated (HCN/ $\mathrm{HCO}^{+} \approx 4$ ) in the high-velocity component of the line, which we attribute to a molecular outflow in the center of the system (Section 3.3). The line ratio in the outflow is approximately the same as the velocity-integrated ratio in the central resolution element, however the line ratio in the central resolution element appears elevated $(>2)$ at all velocities (Figure 6 bottom). This, in addition to the relatively small contribution of the outflow to the total line flux means the HCN enhancement in the center is not solely due to the presence of the outflow and its high $\mathrm{HCN} /$ 

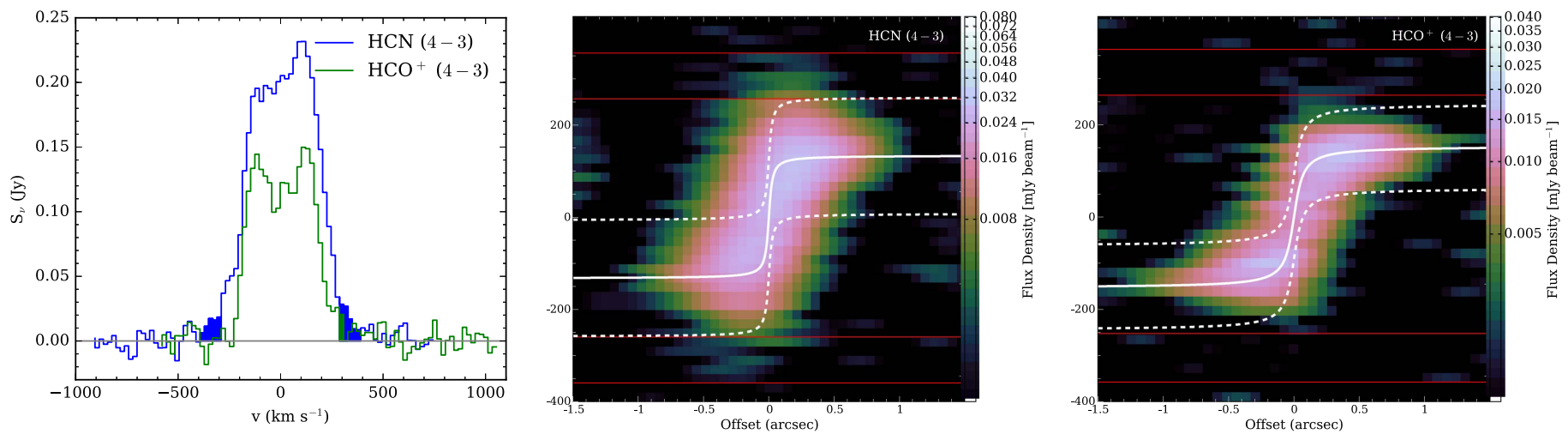

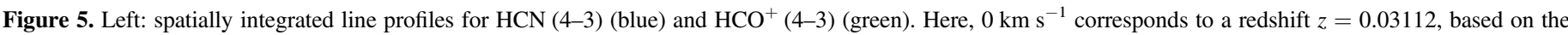

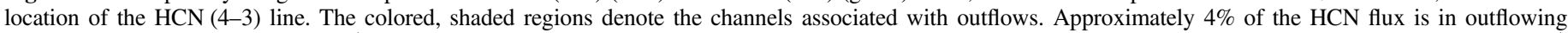

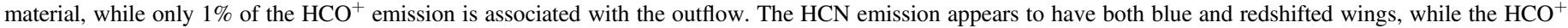

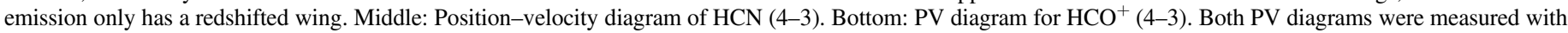

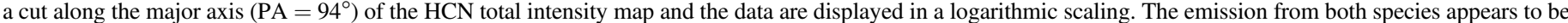

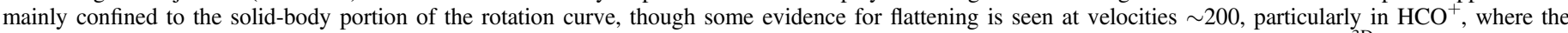

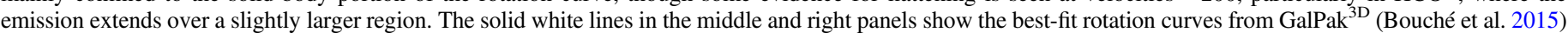

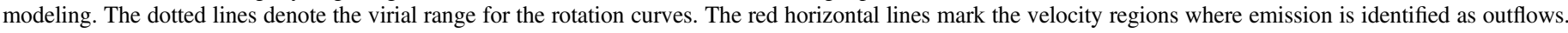
This emission identified as winds lies clearly above the flattening of the rotation curve, suggesting it has a non-rotational component to its velocity.

$\mathrm{HCO}^{+}$ratio. The emission from $\mathrm{HCN}$ is enhanced over that of $\mathrm{HCO}^{+}$at all velocities within the central few hundred parsecs of IRAS 13120-5453.

\section{Water Emission in IRAS 13120-5453}

Figure 7 shows show the Herschel PACS and SPIRE spectra, as well as the spectral line energy distribution of the $\mathrm{H}_{2} \mathrm{O}$ lines detected with SPIRE and PACS. The line fluxes are given in Table 3 . Two of the ten $\mathrm{H}_{2} \mathrm{O}$ transitions targeted by the Hermolirg project were detected in absorption, both with lower level energies of $\lesssim 100 \mathrm{~K}$. We see no obvious contamination by other species in any of the lines. Five $\mathrm{H}_{2} \mathrm{O}$ transitions, with upper level energies of $\lesssim 300 \mathrm{~K}$, were detected in emission with SPIRE.

We have used the spherically symmetric radiative transfer code described by González-Alfonso \& Cernicharo (1997, 1999) to model the observed $\mathrm{H}_{2} \mathrm{O}$ lines and constrain the dust temperature and opacity of the dense ISM. The code includes collisional excitation as well as excitation by the farinfrared field emitted by warm dust. Dust is modeled as a mixture of silicates and amorphous carbon, with an adopted mass absorption coefficient as a function of wavelength which is shown in González-Alfonso et al. (2014).

The models are characterized by the following parameters: the dust opacity at $100 \mu \mathrm{m}\left(\tau_{100}\right)$, the dust temperature $\left(T_{\text {dust }}\right)$, the gas temperature $\left(T_{\text {gas }}\right)$, the $\mathrm{H}_{2}$ density $\left(n_{\mathrm{H}_{2}}\right)$, and the column density of $\mathrm{H}_{2} \mathrm{O}$ per unit velocity dispersion $\left(N_{\mathrm{H}_{2} \mathrm{O}} / \Delta V\right)$. Collisional rates with $\mathrm{H}_{2}$ are taken from Dubernet et al. (2009) and Daniel et al. (2011) for $\mathrm{H}_{2} \mathrm{O}$. We have adopted a gas-to-dust ratio of 100 by mass, guided by the average value in LIRGs reported by Wilson et al. (2008).

Our general approach to the modeling was to compare the observed ratios of various $\mathrm{H}_{2} \mathrm{O}$ lines to a grid of models with varying $T_{\text {dust }}, N_{\mathrm{H}_{2} \mathrm{O}} / \Delta V, \tau_{100}, n_{\mathrm{H}_{2}}$, and $T_{\text {gas }}$. We found that the $\mathrm{H}_{2} \mathrm{O}$ line ratios in IRAS 13120-5453 cannot be reproduced with collisional excitation alone. In fact, the excitation is found to be dominated by absorption of photons emitted by warm dust, with collisions mainly affecting the lowest-lying $\mathrm{H}_{2} \mathrm{O}$ levels. The fact that the $\mathrm{H}_{2} \mathrm{O}_{11} \rightarrow 0_{00}$ line is detected in emission suggests that some collisional excitation should be taken into account. We used $T_{\text {gas }}=150 \mathrm{~K}$ and $n_{\mathrm{H}_{2}}=3 \times 10^{4} \mathrm{~cm}^{-3}$, which yields a thermal pressure similar to that inferred for the warm molecular gas component in Arp 220 (Rangwala et al. 2011). These values are sufficient to produce emission in the $\mathrm{H}_{2} \mathrm{O}_{11} \rightarrow 0_{00}$ but still low enough to leave the higher-lying lines relatively unaffected.

We found that the best fit to the observations is achieved with a dust opacity of $\tau_{100}=0.1-1$, with $\tau_{100}=0.5$ being the preferred value. For these values of $\tau_{100}$ the relative fluxes of the high-lying lines can be well fitted with different combinations of dust temperatures between $T_{\text {dust }}=40$ and $60 \mathrm{~K}$, and $\mathrm{H}_{2} \mathrm{O}$ columns between $N_{\mathrm{H}_{2} \mathrm{O}} / \Delta V=2 \times 10^{14}$ and $5 \times 10^{15} \mathrm{~cm}^{-2}\left(\mathrm{~km} \mathrm{~s}^{-1}\right)^{-1}$. The best fit, which is also included in Figure 7 , is achieved with $\tau_{100}=0.5 T_{\text {dust }}=40 \mathrm{~K}$, and $N_{\mathrm{H}_{2} \mathrm{O}} / \Delta V=2.5 \times 10^{15} \mathrm{~cm}^{-2}\left(\mathrm{~km} \mathrm{~s}^{-1}\right)^{-1}$.

The $\mathrm{H}_{2} \mathrm{O}$ lines are unresolved with Herschel, but it is likely the emission arises from a similar region to the $\mathrm{HCN}$ and $\mathrm{HCO}^{+}$emission (González-Alfonso et al. 2014). Thus, we can use the observed velocity dispersion of those lines as proxy for $\Delta V$ of the $\mathrm{H}_{2} \mathrm{O}$ lines (which are unresolved with Herschel). Taking the mean dispersion of the $\mathrm{HCN}$ and $\mathrm{HCO}^{+}$lines (Figure 8) of $140 \mathrm{~km} \mathrm{~s}^{-1}$ we find a column density of $N_{\mathrm{H}_{2} \mathrm{O}}=3.5 \times 10^{17} \mathrm{~cm}^{-2}$. The $\mathrm{H}_{2}$ column inferred from the best-fit $\tau_{100}$ and our assumed dust-to-gas ratio is $\sim 6.7 \times 10^{23} \mathrm{~cm}^{-2}$. This implies an abundance of $\mathrm{H}_{2} \mathrm{O}$ relative to $\mathrm{H}_{2}$ of $5 \times 10^{-7}$, somewhat lower than what is seen in more compact/obscured systems such as Mrk 231 (GonzálezAlfonso et al. 2008).

We can compare the $\mathrm{H}_{2}$ column derived from the $\mathrm{H}_{2} \mathrm{O}$ modeling with $N_{\mathrm{H}}$ determined from modeling of NuSTAR observations. The NUSTAR $N_{\mathrm{H}}=3.15_{-1.29}^{+2.23} \times 10^{24} \mathrm{~cm}^{-2}$ probes the column between us and the hard X-ray emitting portion of the IRAS 13120-5453, while the Herschel-derived $\mathrm{H}_{2}$ column probes the entire line of sight through the nucleus (assuming the $\mathrm{H}_{2} \mathrm{O}$ emission traces the entire ISM). If the ISM is symmetrically distributed about the nucleus, approximately half of the $\sim 6.7 \times 10^{23} \mathrm{~cm}^{-2} \mathrm{H}_{2}$ is between us and the nucleus and the other half is on the far side of the Galaxy. Considering that the absorption cross-section of the hydrogen atoms is not significantly affected by being bound in molecules (Cruddace 

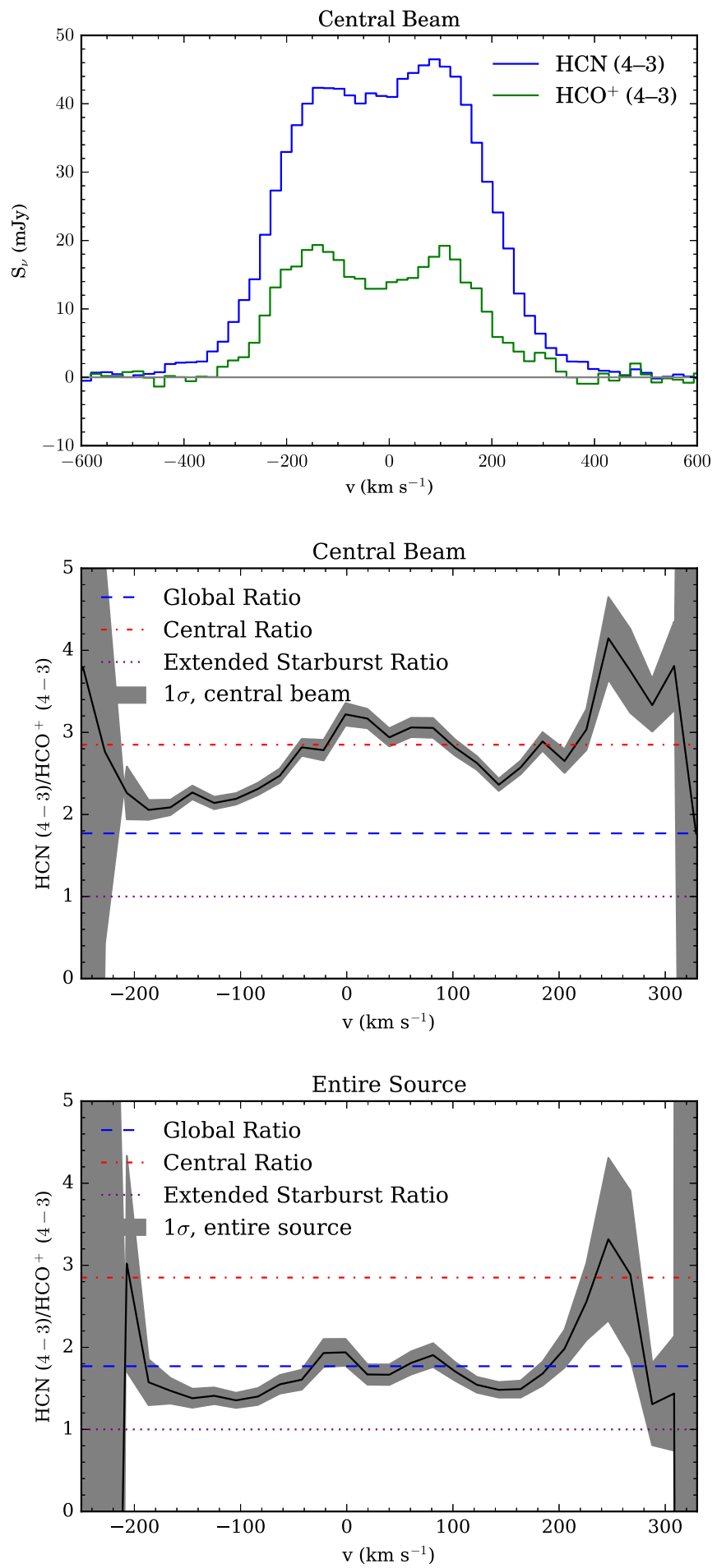

Figure 6. Top: the $\mathrm{HCN}(4-3)$ and $\mathrm{HCO}^{+}(4-3)$ line profiles in the central resolution element. Middle: the $\mathrm{HCN} / \mathrm{HCO}^{+}$ratio as a function of velocity, for the central resolution element (black line). Bottom: the $\mathrm{HCN} / \mathrm{HCO}^{+}$ratio as a function of velocity, integrated over the entire source (black line). In the lower two panels, the shaded region marks the $1 \sigma$ statistical uncertainty in the velocity-resolved ratio. For comparison we show the velocity-integrated line ratio for the entire source $(1.77$ blue dashed line) and for the central resolution element (2.85; red dotted-dashed line). The ratio is further enhanced in the channels associated with outflowing gas (Section 3.3). The line ratios near the systemic velocity may be affected by selfabsorption, which appears to impact $\mathrm{HCO}^{+}$(4-3) more strongly than $\mathrm{HCN}$ (4-3).

et al. 1974; Morrison \& McCammon 1983), the offsetting factors of two imply the molecular ISM could contribute $N_{\mathrm{H}, \text { mol }} \sim 6.7 \times 10^{23} \mathrm{~cm}^{-2}$, or $\sim 20 \%$ of the AGN's obscuring
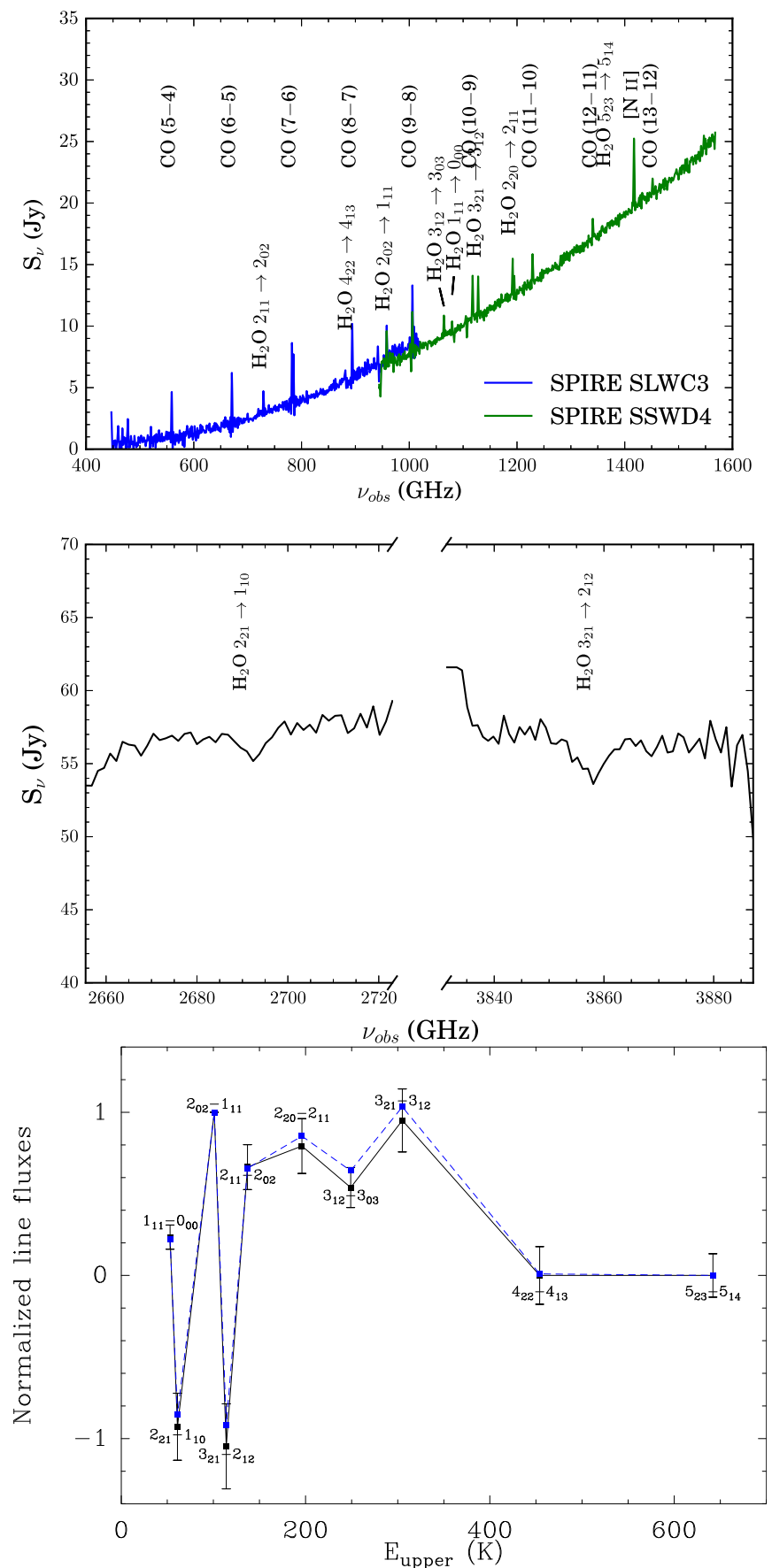

Figure 7. Top: Herschel SPIRE spectrum of IRAS 13120-5453, with the locations of [N II], $\mathrm{CO}$, and $\mathrm{H}_{2} \mathrm{O}$ lines marked. Presentation of the $\mathrm{CO}$ and [N II] lines can be found in Kamenetzky et al. (2016) and N. Lu et al. (2016, in preparation). Middle: Herschel PACS spectrum, with the locations of $\mathrm{H}_{2} \mathrm{O}$ lines marked. See Table 3 for measured fluxes of the $\mathrm{H}_{2} \mathrm{O}$ lines and Figure 7 for the $\mathrm{H}_{2} \mathrm{O}$ SLED. Bottom: spectral line energy distribution of the $\mathrm{H}_{2} \mathrm{O}$ lines detected with SPIRE and PACS. The black squares present the data, normalized to the flux of the $\mathrm{H}_{2} \mathrm{O} 2_{02} \rightarrow 1_{11}$ line. The best fit model is shown as a dashed blue line.

column. The molecular ISM may be a significant contributor to the Compton-thick screen between us and the AGN in IRAS $13120-5453$.

We note the dust temperature derived from modeling of the $\mathrm{H}_{2} \mathrm{O}$ lines does not provide constraints on the possibility of infrared pumping of $\mathrm{HCN}$ as it reflects the dust temperature of the overall nuclear ISM, rather than any compact (10s of pc) 
Table 3

$\mathrm{H}_{2} \mathrm{O}$ Lines Detected with Herschel

\begin{tabular}{|c|c|c|c|c|}
\hline Line & $\begin{array}{c}\nu_{\text {rest }} \\
(\mathrm{GHz})\end{array}$ & $\begin{array}{c}E_{\text {upper }} \\
(\mathrm{K})\end{array}$ & $\begin{array}{l}\text { Cont. }^{\mathrm{a}} \\
\text { (Jy) }\end{array}$ & $\begin{array}{c}\text { Flux } \\
\left(\mathrm{Jy} \mathrm{km} \mathrm{s}^{-1}\right)\end{array}$ \\
\hline $\mathrm{H}_{2} \mathrm{O} \mathrm{l}_{11} \rightarrow 0_{00}$ & 1113.34 & 53 & 9.6 & $314.2 \pm 81.0$ \\
\hline $\mathrm{H}_{2} \mathrm{O} 2_{02} \rightarrow 1_{11}$ & 987.93 & 101 & 6.8 & $1337.5 \pm 244.3$ \\
\hline $\mathrm{H}_{2} \mathrm{O} 2_{11} \rightarrow 2_{02}$ & 752.03 & 137 & 2.9 & $888.1 \pm 86.2$ \\
\hline $\mathrm{H}_{2} \mathrm{O} 2_{20} \rightarrow 2_{11}$ & 1228.79 & 196 & 12.6 & $1061.3 \pm 113.9$ \\
\hline $\mathrm{H}_{2} \mathrm{O} 3_{12} \rightarrow 3_{03}$ & 1097.36 & 249 & 9.2 & $720.8 \pm 98.2$ \\
\hline $\mathrm{H}_{2} \mathrm{O} 3_{21} \rightarrow 3_{12}$ & 1162.91 & 305 & 10.8 & $1271.1 \pm 113.3$ \\
\hline $\mathrm{H}_{2} \mathrm{O} 4_{22} \rightarrow 4_{13}$ & 916.17 & 454 & 12.0 & $<236.3$ \\
\hline $\mathrm{H}_{2} \mathrm{O} 5_{23} \rightarrow 5_{14}$ & 1410.62 & 642 & 18.0 & $<177.8$ \\
\hline $\mathrm{H}_{2} \mathrm{O}_{21} \rightarrow 2_{12}$ & 3977.05 & 114 & 56.7 & $-1400.7 \pm 237$ \\
\hline $\mathrm{H}_{2} \mathrm{O} 2_{21} \rightarrow 1_{10}$ & 2773.98 & 61 & 57.1 & $-1240.3 \pm 156$ \\
\hline
\end{tabular}

Note.

${ }^{\mathrm{a}}$ Value of the fitted baseline at the line center.

hot nuclear core (though such a core appears unlikely to be present, based on the lack of detectable IR pumping and the low $\Sigma_{14}$; Section 3.2.1).

\section{Nuclear Kinematics}

In Figure 8 we show the intensity-weighted velocity field (moment 1; top row) and velocity dispersion (moment 2; bottom row) for $\mathrm{HCN}$ (4-3) (left) and $\mathrm{HCO}^{+}$(4-3) (right). The emission from both species appears broadly consistent with a rotating disk, with centrally peaked velocity dispersion.

Correspondingly, the PV diagrams taken along the observed major axis of the emission (Figure 5) show clear signatures of rotation. The (4-3) emission from both species appears to be mainly concentrated in the solid-body rotation portion of the potential, though the $\mathrm{HCO}^{+}$(4-3) velocity begins to flatten out beyond $\sim 0$ !" $75(0.48 \mathrm{kpc})$. The emission tentatively identified as the outflow lies above the apparent turnover in the rotation curve and outside the estimated virial range, consistent with a scenario in which the gas motion is not solely due to the gravitational potential.

We adopt $z=0.03112$ as the systemic redshift, based on the moment 1 value at the center of the detected ALMA continuum emission $\left(13^{\mathrm{h}} 15^{\mathrm{m}} 06^{\mathrm{s}} .32,-55^{\mathrm{d}} 09^{\mathrm{m}} 22.78\right)$. This is somewhat higher than the optical redshift found on $\operatorname{NED}(z=0.030761)$ as measured by Strauss et al. (1992). The optical redshift was determined using $\mathrm{H} \alpha$, and so could be somewhat offset from the true systemic by obscuration.

\subsection{Nuclear Dynamical Mass}

Using both the $\mathrm{HCN}(4-3)$ and $\mathrm{HCO}^{+}$(4-3) emission, we can estimate the dynamical mass as $M \sim V_{\text {circ }}^{2} R / G$. Within a $1 \mathrm{kpc}$ diameter region, $\mathrm{HCN}$ and $\mathrm{HCO}^{+}$have FWHMs of $385 \mathrm{~km} \mathrm{~s}^{-1}$ and $375 \mathrm{~km} \mathrm{~s}^{-1}$, respectively and we compute the circular velocity as $V_{\text {circ }}=\mathrm{FWHM} /(2 \sqrt{\ln 2})$. This corresponds to estimated dynamical masses of $1.2(\sin i)^{-2} \times$ $10^{10} M_{\odot}$ and $1.0(\sin i)^{-2} \times 10^{10} M_{\odot}$. Adopting the mean value of $1.1(\sin i)^{-2} \times 10^{10} M_{\odot}$, and the area $\left(0.78 \mathrm{kpc}^{-2}\right)$, we find a total mean mass surface density of $1.4(\sin i)^{-2} \times$ $10^{10} M_{\odot} \mathrm{kpc}^{-2}$. Assuming the disk is intrinsically circular, the observed axis ratios suggest $i \approx 55^{\circ}$, leading to an inferred dynamical mass of $1.6 \times 10^{10} M_{\odot}$ and a mean mass surface density of $2.0 \times 10^{10} M_{\odot} \mathrm{kpc}^{-2}$ within the central kpc.

\section{Nuclear Continuum Emission}

We detect $333 \mathrm{GHz}$ continuum emission with a total flux of $89.8 \pm 0.4 \mathrm{mJy}$. For this flux, and $T_{\text {dust }}=40 \mathrm{~K}$ derived from the $\mathrm{H}_{2} \mathrm{O}$ modeling, and a mass absorption coefficient of the dust at $333 \mathrm{GHz}$ of $\kappa(333 \mathrm{GHz})=0.28 \mathrm{~cm}^{2} \mathrm{~g}^{-1}$ (consistent with Milky Way dust properties; Bianchi 2013); this implies a dust mass of $2.9 \times 10^{8} M_{\odot}$. If we assume a gas-to-dust ratio of 100 (Wilson et al. 2008), the ISM mass is then $\sim 3 \times 10^{10} M_{\odot}$. Alternately, using the empirical $L_{850 \mu \mathrm{m}}-M_{\text {ISM }}$ relation derived from low-z galaxies (Scoville et al. 2014), the continuum emission implies a similar total ISM mass of $(3.2 \pm 0.7) \times$ $10^{10} M_{\odot \cdot}{ }^{17}$

The detected continuum emission is contained within an ellipse with axes of 2 !. $5 \times 1$ ".9 $(1.6 \mathrm{kpc} \times 1.2 \mathrm{kpc}$; Figure 9). The distribution of the emission is well represented by a 2D Gaussian with a beam-deconvolved size (FWHM) of 0 ." $86 \times 0$. . $75(0.56 \mathrm{kpc} \times 0.49 \mathrm{kpc})$ at a PA of $60^{\circ}$, and is marginally resolved. This is the best measurement for the size of the nuclear starburst in IRAS 13120-5453, improving the constraints on the area of the starburst by a factor of $\sim 25$.

Díaz-Santos et al. (2010) estimated that $\sim 80 \%$ of $L_{\mathrm{IR}}$ originates in the compact starburst. If we assume $L_{\mathrm{IR}}$ is distributed in the same way as the submillimeter continuum emission, we can estimate the IR luminosity surface density within the half-light radius as $\Sigma_{\mathrm{IR}, 50}=L_{\mathrm{IR}, 50} / A_{50}$, where $L_{\mathrm{IR}, 50}$ is the luminosity contained within the half-light radius of the nuclear starburst $\left(0.4 L_{\mathrm{IR}}\right.$, considering only the unresolved portion) and $A_{50}=\pi a_{\mathrm{sb}} b_{\mathrm{sb}}$ is the half-light area with semimajor and semiminor axes of $a_{\mathrm{sb}}$ and $b_{\text {sb. }}$. We find $\Sigma_{\mathrm{IR}, 50}=4.7 \times 10^{12} L_{\odot} \mathrm{kpc}^{-2}$. This is $\sim 10 \times$ larger than the $\Sigma_{\mathrm{IR}}$ inferred by Díaz-Santos et al. (2010) based on Spitzer data. Compared to the [C II] deficit relation for starbursts from Díaz-Santos et al. (2014), our $\Sigma_{\mathrm{IR}, 50}$ places IRAS 13120-5453 on the correlation of the [C II] deficit and starburst luminosity surface density, suggesting the [C II] deficit in IRAS 13120-5453 can be mostly explained by the compact starburst and does not require significant AGN contribution to $L_{\mathrm{FIR}}$. Using the same size, we find an ISM surface density of $\Sigma_{\text {ISM,50 }}=5.7 \times 10^{10} M_{\odot} \mathrm{kpc}^{-2}$ within the half-light radius.

Within a $1 \mathrm{kpc}$ diameter, the $333 \mathrm{GHz}$ continuum emission is consistent with an ISM mass of $(1.4 \pm 0.4) \times 10^{10} M_{\odot}$ and a corresponding mass surface density of $\Sigma_{\mathrm{ISM}, 1 \mathrm{kpc}}=$ $(1.7 \pm 0.5) \times 10^{10} M_{\odot} \mathrm{kpc}^{-2}$. This is comparable to the dynamical mass estimated from the $\mathrm{HCN}$ and $\mathrm{HCO}^{+}$ kinematics (Section 5.1) and implies $M_{\mathrm{ISM}} / M_{\mathrm{dyn}} \approx 0.9$ within the central kpc. It is possible the ISM mass estimate from these ALMA data, which use the empirical calibration of Scoville et al. (2014), are biased high, relative to the galaxies used to calibrate it if the mass-weighted dust dust temperature is higher in IRAS 13120-5453. The empirical calibration was arrived at using a galactic conversion factor between $L_{\mathrm{CO}}^{\prime}$ and $\mathrm{M}_{\mathrm{H}_{2}}$-if the conversion factor varies from this value in the galaxies used

\footnotetext{
17 We note the Scoville et al. (2014) calibration includes the H I mass and assumes this mass of this atomic ISM component is equal to $50 \%$ of the molecular mass. The Scoville et al. (2016) calibration does not consider the $\mathrm{H} \mathrm{I}$ mass, so the empirical normalization is reduced by $1 / 3$. Thus, to obtain only the molecular ISM mass (equivalent to the Scoville et al. 2016) calibration, ISM mass derived directly from the continuum flux should be multiplied by 0.67 .
} 

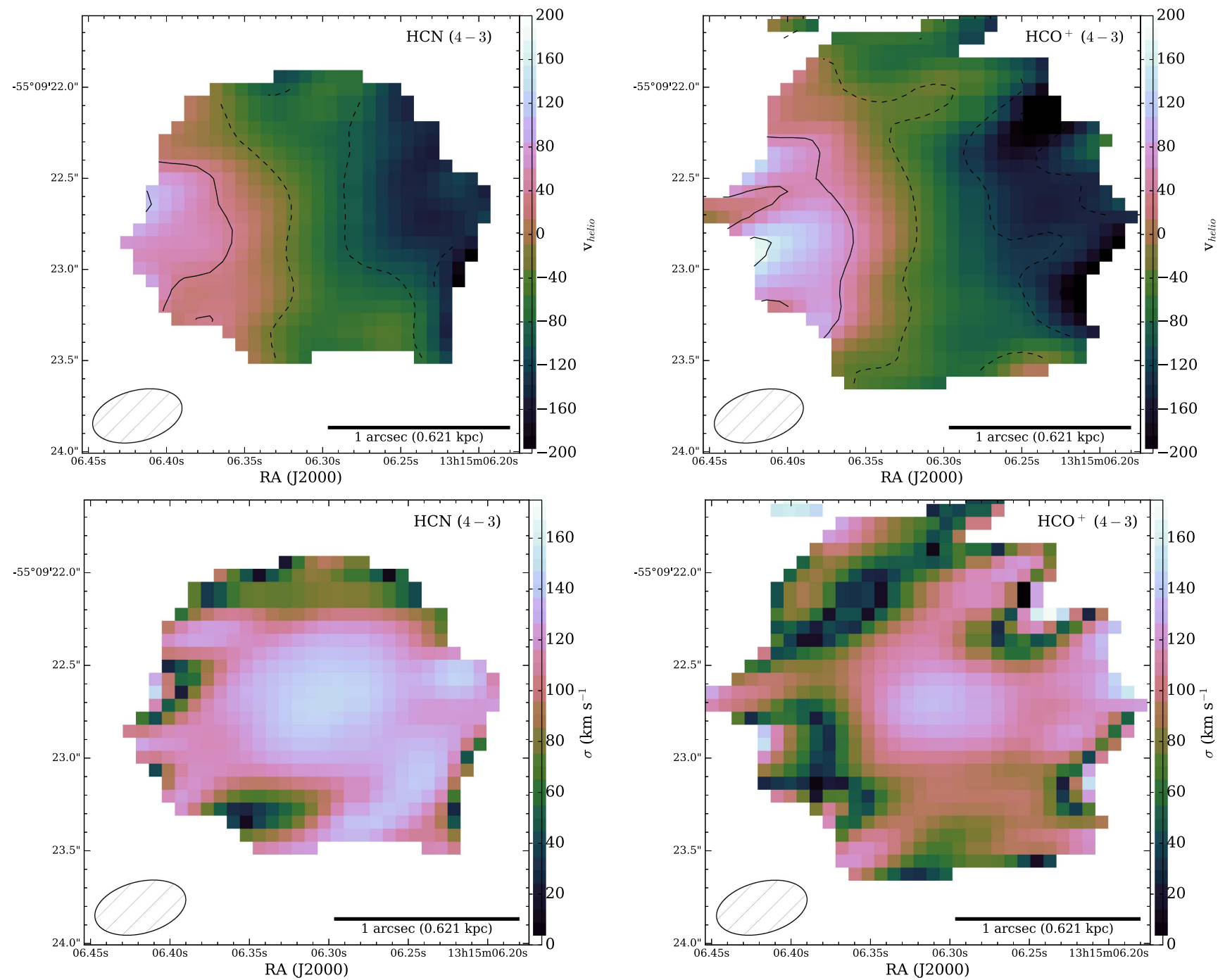

Figure 8. Top row: intensity-weighted velocity map for $\mathrm{HCN}(4-3)$ (left) and $\mathrm{HCO}^{+}(4-3)$ (right). Contours are spaced every $50 \mathrm{~km} \mathrm{~s}{ }^{-1}$. Bottom row: intensityweighted velocity dispersion map (moment 2 ) for $\mathrm{HCN}$ (4-3) (left) and $\mathrm{HCO}^{+}$(4-3) (right). The extent of each map was determined by masking the datacube at the $3 \sigma$ level in the corresponding total intensity (moment 0 ) map. The velocity fields of both molecules are consistent with ordered rotation, in a $\sim 1 \mathrm{kpc}$ central molecular disk. Both species show centrally peaked velocity dispersions, though the $\mathrm{HCO}^{+}$peaks at a slightly lower value.

to calibrate the relation, the resulting empirical relationship would overestimate the ISM mass.

Alternately, the gas-to-dust ratio may be different in IRAS 13120-5453, compared to the calibration sources in Scoville et al. (2014). Wilson et al. (2008) found a mean gas-to-dust ratio of 120 for IR-luminous galaxies, but there is an order of magnitude range in the gas-to-dust ratio for the systems in that study $((29 \pm 8)-(725 \pm 286))$. If the gas-to-dust ratio in IRAS $13120-5453$ is on the lower end of the range, the actual ISM mass would be correspondingly lower and in less tension with the dynamical mass. Furthermore, our ISM mass estimate calibration includes an assumed H I component; if we instead adopt the calibration of Scoville et al. (2016) for only the molecular ISM, we would find a value reduced by $1 / 3$. For comparison, the nuclear gas fraction in systems such as Arp 220 and NGC 6240 are $\sim 1 / 3$ (Scoville et al. 1997; Solomon et al. 1997; Sakamoto et al. 1999; Downes \& Eckart 2007; Scoville et al. 2015), showing that $M_{\text {ISM }}$ is a substantial fraction of $M_{\text {dyn }}$ in ULIRGs. If we assume IRAS $13120-5453$ has a similar gas fraction, the implied gas-to-dust ratio would be on the order of 30 or 40 , consistent with the range of Wilson et al. (2008), but on the lower end. Finally, the ISM mass estimate derived from the dust mass is sensitive to the choice of $\kappa$. An elevated value of $\kappa$ would reduce the calculated dust mass and the inferred ISM mass.

We can make an additional estimate of the ISM mass from the $\mathrm{H}_{2}$ column density, $N_{\mathrm{H}}$, inferred from the $\mathrm{H}_{2} \mathrm{O}$ modeling (Section 4) as $M_{\mathrm{ISM}}=\mu(4 / 3) \pi R^{2} m_{\mathrm{H}} N_{\mathrm{H}}$, where $\mu=1.4$ accounts for the mass in helium, $R$ is the radius, $m_{\mathrm{H}}$ is the mass of hydrogen. For $R=500 \mathrm{pc}$, we calculate $M_{\mathrm{ISM}}=$ $7.5 \times 10^{9}$ and an implied $M_{\mathrm{ISM}} / M_{\mathrm{dyn}}=0.47$. This ISM mass estimate is sensitive to the best-fit dust temperature and $\tau_{100}$ inferred from the $\mathrm{H}_{2} \mathrm{O}$ modeling. Higher temperatures and lower $\tau_{100}$ values would further reduce the estimate ISM mass.

Using the starburst size, inferred $\Sigma_{\mathrm{IR}, 50}$, and gas fraction of $f_{g} \approx 0.3$ (as observed in sources with similar $L_{\mathrm{IR}}$ ), we can compare with the Thompson et al. (2005) model for a radiation pressure-limited starburst. We find that IRAS $13120-5453$ is near to but slightly below the maximal starburst line for these values (for $\sigma \approx 200 \mathrm{~km} \mathrm{~s}^{-1}$ ).

For the measured peak flux density of $12.6 \mathrm{mJy}$ beam $^{-1}$ the brightness temperature at $333 \mathrm{GHz}$ is $2.7 \mathrm{~K}$, suggesting the dust 


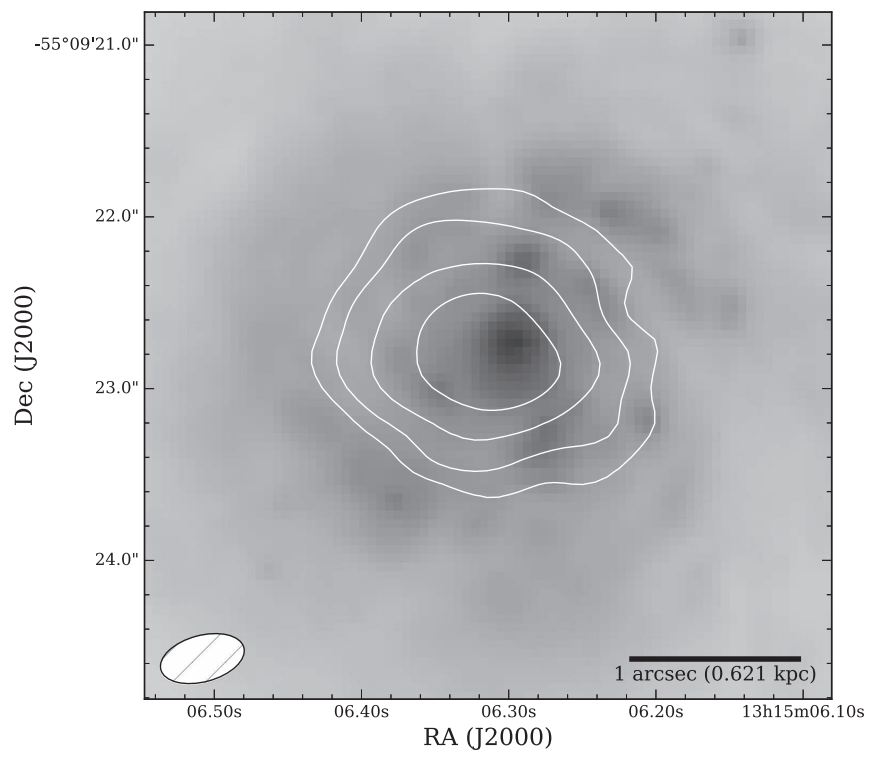

Figure 9. Map of the $333 \mathrm{GHz}$ continuum emission (white contours), superimposed on an HST/ACS F814W image of IRAS 13120-5453. The contour levels are $1,2,4$, and $8 \mathrm{mJy}_{\text {beam }}{ }^{-1}$. The relative astrometry of the $H S T /$ ACS image is uncertain to roughly $1^{\prime \prime}$, so the $0.8 \mathrm{~mm}$ continuum peak is consistent with the position of the optical nucleus.

emission on the scale of our beam is optically thin. If we take the $40 \mathrm{~K}$ dust temperature from modeling of the $\mathrm{H}_{2} \mathrm{O}$ lines as the true dust temperature, we estimate $\tau=0.071$.

\subsection{Comparison to Planck Limit and Herschel Extrapolation}

Our ALMA detection is consistent with the Planck $357 \mathrm{GHz}$ non-detection, which reported an upper limit of $\sim 200 \mathrm{mJy}$ (Planck Collaboration et al. 2014). For an additional estimate of the total $333 \mathrm{GHz}$ continuum emission we fit the Herschel PACS and SPIRE photometry (J. Chu et al. 2016, in preparation) with a single-component modified blackbody. We fix the dust emissivity to $\beta=1.8$ (e.g., Planck Collaboration et al. 2011). and derive a best-fit temperature, $T_{\mathrm{BB}}=$ $33 \pm 0.5 \mathrm{~K} .{ }^{18}$ Using this modified blackbody fit we predict a $333 \mathrm{GHz}$ flux of $\sim 190 \mathrm{mJy}$ (Figure 10, top).

If this extrapolation is correct, it suggests were are resolving out up to $\sim 50 \%$ of the $333 \mathrm{GHz}$ flux with our ALMA observations. This missing flux should be extended on scales $>4^{\prime \prime}(>2.6 \mathrm{kpc}$; the scale on which our observations will be affected by spatial filtering $)$ and $<36$ "' $2(<23.7 \mathrm{kpc}$; the SPIRE beam at $500 \mu \mathrm{m})$, since we are using galaxy-integrated values. ${ }^{19}$ This deficit of flux corresponds to an additional $M_{\text {ISM }} \approx 3.2 \times 10^{10} M_{\odot}$ (following the Scoville et al. 2014 empirical relation). Taking $4^{\prime \prime}$ as a lower-limit on the scale for all of the missing flux, this implies the $\Sigma_{\text {ISM }}$ of any missing, extended component must be $<10^{9} M_{\odot} \mathrm{kpc}^{-2}$. This is at least a factor of 10 lower than the mass determined for the inner kpc, so our estimate of $\Sigma_{\mathrm{ISM}, 1 \mathrm{kpc}}$ is unlikely to be strongly biased by missing continuum flux, and uncertainties our estimates of the ISM mass surface density are likely dominated by the scatter in the $M_{\mathrm{ISM}}-L_{850 \mu \mathrm{m}}$ relation.

\footnotetext{
${ }^{18}$ Errors obtained using MCMC exploration with the emcee package (Foreman-Mackey et al. 2013) and represent the $1 \sigma$ range.

19 We note that IRAS 13120-5453 is unresolved in all the Herschel PACS and SPIRE bands, including the $70 \mu \mathrm{m}$ band, which has a resolution of 5.6 $(3.7 \mathrm{kpc})$.
}
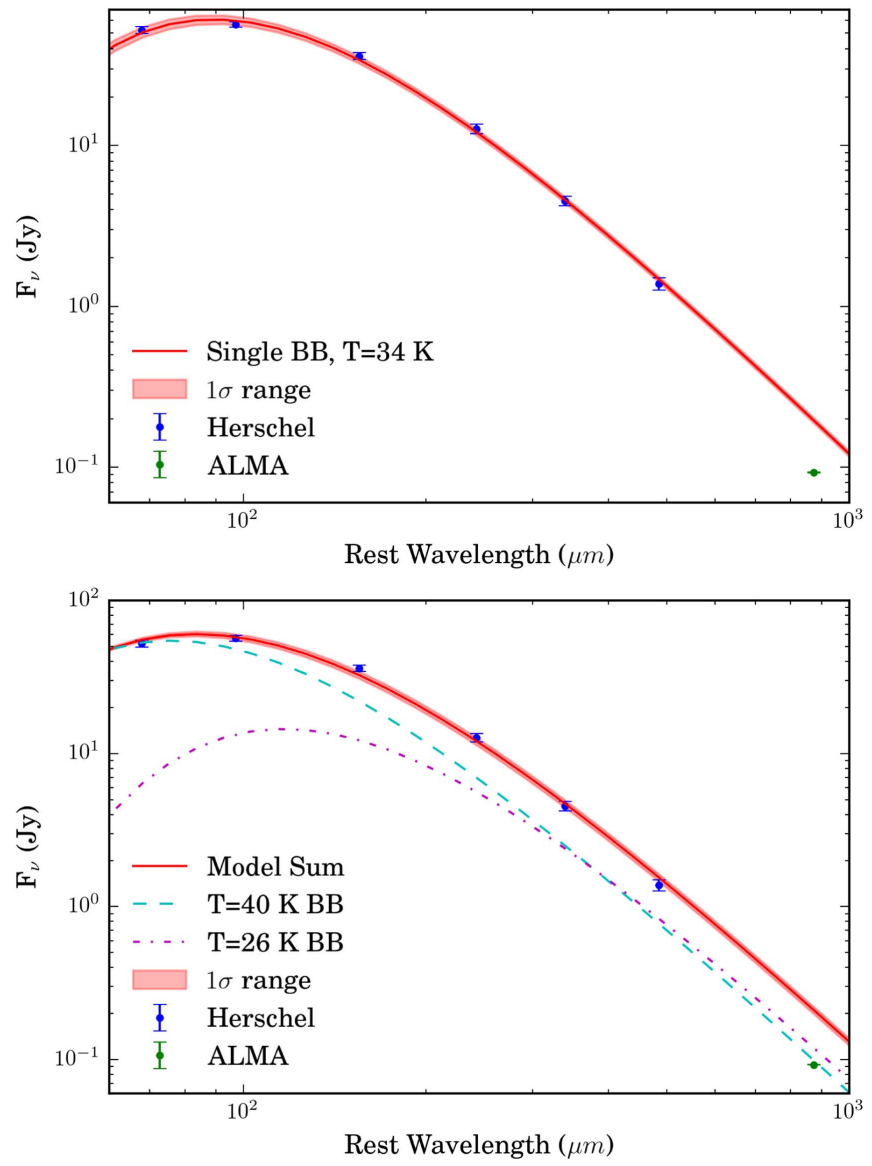

Figure 10. Herschel PACS and SPIRE spectral energy distribution of IRAS 13120-5453 (fluxes from J. Chu et al. 2016, in preparation), fit with two different models. Top: single-component modified blackbody fit with a best-fit temperature of $34 \mathrm{~K}$. Bottom: two-component modified blackbody fit. One component was fixed in temperature to $40 \mathrm{~K}$, as derived from our $\mathrm{H}_{2} \mathrm{O}$ modeling and the amplitude fixed to that of the ALMA $333 \mathrm{GHz}$ flux measurement. The temperature and amplitude of the second component was left free and the sum was fit to the Herschel measurements. The best-fit temperature for the second component is $26 \mathrm{~K}$. For all modified blackbodies we fix the dust emissivity to $\beta=1.8$. Both fits to the Herschel photometry predict a similar $333 \mathrm{GHz}$ flux density, $190 \mathrm{mJy}$ from the single-component and 210 mJy for the two-component model. Both predictions are broadly consistent with the Planck upper limit (Planck Collaboration et al. 2014) and would suggest that these ALMA observations are resolving out approximately half the flux, which would be extended on scales $>4^{\prime \prime}$.

Using the Herschel-derived temperature and the extrapolated flux, the dust mass would be $M_{\text {dust,extrapolated }}=8.2 \times 10^{8} M_{\odot}$. Assuming a gas-to-dust ratio of 100 implies an ISM mass of $M_{\mathrm{ISM} \text {,extrapolated }}=8 \times 10^{10} M_{\odot}$, a factor of 2.6 higher than inferred from our ALMA observations.

The dust temperature inferred from the Herschel photometry ( $34 \mathrm{~K}$ ) is lower than that obtained from the modeling of $\mathrm{H}_{2} \mathrm{O}$ lines $(40 \mathrm{~K})$. This is likely due to the fact that the $\mathrm{H}_{2} \mathrm{O}$ lines are tracing the denser molecular gas while the Herschel continuum measurements with a larger beam and fewer spatial filtering issues likely include emission from cooler, diffuse dust.

To further explore this, we also fit the Herschel measurements with a two-component model, consisting of a $40 \mathrm{~K}$ modified blackbody normalized to our measured ALMA flux and a second modified blackbody with the temperature and normalization left as free parameters. For both components, we left $\beta$ fixed to 1.8. This two-component model predicts a 
$333 \mathrm{GHz}$ flux of $\sim 210 \mathrm{mJy}$ (Figure 10, bottom), similar to that of the single-component model and roughly consistent with the Planck upper limit.

The best-fit temperature of the second blackbody component is $26 \mathrm{~K}$. This is consistent with dust temperatures for normal galaxies (e.g., Skibba et al. 2011). Because the $40 \mathrm{~K}$ blackbody was normalized to the integrated ALMA flux, the excess flux from the $26 \mathrm{~K}$ blackbody, if present and resolved out by our ALMA observations, should be extended on scales $>4^{\prime \prime}$. This two-component model is thus consistent with a scenario in which (U)LIRGs have concentrated nuclear starbursts which are surrounded by less intense star formation that more closely resembles normal galaxies. This is consistent with the results of Díaz-Santos et al. (2014), who found the extended [C II] emission of IR-selected galaxies to be similar to normal starforming galaxies, even if the nuclear starbursts show pronounced [C II] deficits. In the Díaz-Santos et al. (2014) sample, this extended star formation was detected on scales of $1-12.6 \mathrm{kpc}$, and thus the scale of this diffuse star formation is comparable to the physical scale of any flux resolved out by our ALMA observations $(2.6 \mathrm{kpc})$.

We note that the $40 \mathrm{~K}$ blackbody, anchored to the ALMA data-point, falls below the observed $14 \mu \mathrm{m}$ flux (Inami et al. 2013), meaning an additional higher-temperature component would be needed to fit the mid-infrared portion of the SED, though the same is true for the single-component $T=34 \mathrm{~K}$ fit to the Herschel data.

We prefer the two-component fit to the Herschel bands over the single-component fit owing to its more realistic representation of the likely physical origin of the thermal dust emission in IRAS 13120-5453. The $40 \mathrm{~K}$ component dominates the IR luminosity of the system (Figure 10, bottom), thus we conclude the $333 \mathrm{GHz}$ emission is a reasonable proxy for the distribution of the nuclear starburst containing $\sim 80 \%$ of $L_{\mathrm{IR}}$ and that the size offers a reliable way to estimate $\Sigma_{\mathrm{IR}}$. Continuum observations at $\sim 333 \mathrm{GHz}$ with a single-dish telescope or ALMA in a compact configuration would provide a reliable measurement of the total flux at this frequency and a definitive statement on how much flux is resolved out with our observations. In addition, ALMA observations would afford a measure of distribution of any flux missing in these observations.

\section{The Dense Molecular Gas and the Nuclear Activity in IRAS 13120-5453}

The submillimeter continuum emission tracing the starburst has a diameter of $\sim 500 \mathrm{pc}$ (FWHM from the Gaussian fit). This area of the starburst is associated with an elevated HCN (4-3)/ $\mathrm{HCO}^{+}$(4-3) ratio, and there is tentative evidence for a dense molecular wind. What do these observations imply about the molecular gas properties and the nuclear activity in IRAS 13120-5453?

\subsection{The Excitation of the Dense Molecular Gas}

Of the physical processes discussed in Section 1.1 that could plausibly lead to enhanced $\mathrm{HCN} / \mathrm{HCO}^{+}$emission, radiative pumping seems unlikely due to the lack of detectable $\mathrm{HCN}$ $v_{2}=1 f$ emission. Previous studies of global $\mathrm{HCN} / \mathrm{HCO}^{+}$ ratios in IR galaxies have found limited evidence to support XDRs as the driver of enhanced HCN emission (Costagliola et al. 2011; Privon et al. 2015), and source compactness (traced by $\left.[\mathrm{C} \mathrm{II}] / L_{\mathrm{FIR}}\right)$ is only weakly correlated with $\mathrm{HCN}$ enhancements (Privon et al. 2015). While we cannot model the conditions of the dense molecular gas in IRAS 13120-5453 with the limited set of lines presented here, exploratory LVG modeling has suggested enhanced $\mathrm{HCN}$ abundances may be required to explain the observed $\mathrm{HCN} / \mathrm{HCO}^{+}$ratio over the nucleus. Several recent studies have proposed mechanical heating can explain HCN enhancements (Loenen et al. 2008; Kazandjian et al. 2012; Izumi et al. 2016); can mechanical heating explain this abundance enhancement in IRAS 13120-5453?

We can use the measured star formation properties to estimate the supernova rate and the associated mechanical heating rate in the starburst of IRAS 13120-5453. The star formation rate of $170 M_{\odot} \mathrm{yr}^{-1}$ implies a supernova rate of approximately $1.2 \mathrm{yr}^{-1}$ (for a Salpeter IMF; Mattila \& Meikle 2001), and an associated injection of mechanical energy via shocks. This star formation rate is mostly contained within the region of elevated $\mathrm{HCN} / \mathrm{HCO}^{+}$. We similarly expect the supernovae to be concentrated within this region and so associated with the elevated HCN emission. Using $40 \%$ of the supernova rate estimated above (to match the fraction of $L_{\mathrm{IR}}$ expected to be contained within the starburst FWHM) and the size of the starburst from the millimeter continuum emission $\left(d_{\mathrm{SB}}=500 \mathrm{pc}\right.$; Section 6), and Equation (1) from Kazandjian et al. (2012), we estimate the mechanical energy injection from supernovae to be $\Gamma_{\text {mech }}>8 \times 10^{-21} \mathrm{erg} \mathrm{s}^{-1} \mathrm{~cm}^{-3}$, for a PDR volume filling factor of 0.1 . At this level of $\Gamma_{\text {mech }}$ and above, the Kazandjian et al. (2012) models of mechanical heating in molecular clouds show substantial abundance enhancements of $\mathrm{HCN}$ relative to $\mathrm{HCO}^{+}(3-300 \times)$ and are sufficient to explain the ratios we see here, and are consistent with our exploratory LVG modeling.

Significant non-gravitational motion of the gas would also serve to increase the turbulence in the dense gas disk, which has $\sigma \gtrsim 100 \mathrm{~km} \mathrm{~s}^{-1}$ (Figure 8). We also note that the observed outflow/wind, can also result in mechanical heating (e.g., Izumi et al. 2016), so the molecular wind seen here and by Veilleux et al. (2013) may further increase the mechanical heating rate above that from the supernovae.

The majority of systems with substantial [C II] deficits observed by González-Alfonso et al. (2015) show evidence for strong OH $65 \mu \mathrm{m}$ absorption; IRAS $13120-5453$ is an outlier in this respect, with a significant [C II] deficit but no observed OH $65 \mu \mathrm{m}$ absorption. González-Alfonso et al. (2015) argue this $\mathrm{OH} 65 \mu \mathrm{m}$ absorption requires warm dust $(T>50 \mathrm{~K})$ and high column densities $\left(N_{\mathrm{H}} \gtrsim 6 \times 10^{23} \mathrm{~cm}^{-2}\right)$. Thus, the lack of $\mathrm{OH} 65 \mu \mathrm{m}$ absorption suggests the dust is cooler and/or the column densities are somewhat low, consistent with the nondetection of $\mathrm{H}_{2} \mathrm{O}$ lines above $400 \mathrm{~K}$, the modeling results for the $\mathrm{H}_{2} \mathrm{O}$ lines (Section 4), and the non-detection of the $v_{2}=1 f$ $\mathrm{HCN}$ (4-3) line. The dust temperature of $40 \mathrm{~K}$ obtained from modeling of the $\mathrm{H}_{2} \mathrm{O}$ lines is consistent with the non-detection of $\mathrm{OH} 65 \mu \mathrm{m}$ absorption.

The general role of infrared pumping in enhancing $v_{2}=0$ lines is still uncertain, but our non-detection of the $v_{2}=1 f$ $\mathrm{HCN}$ (4-3) line and the non-detection of OH65 suggest that the starburst in IRAS 13120-5453 is too cool and/or has insufficient column density for $\mathrm{HCN}$ to absorb significant numbers of IR photons. Though not conclusive, this suggests that radiative pumping is not a significant contributor to the enhanced $\mathrm{HCN}$ emission. 
These observations and associated studies of IRAS 13120-5453 are consistent with a scenario in which the molecular gas is primarily experiencing collisional excitation with the enhanced $\mathrm{HCN}$ emission originating from an elevated abundance caused by mechanical heating from the ongoing starburst. Confirmation of the abundance enhancement in the nucleus of IRAS 13120-5453 will require new multi-transition $\mathrm{HCN}$ and $\mathrm{HCO}^{+}$maps to facilitate more detailed LVG modeling.

In contrast to the nuclear regions, the extended regions $\left(\gtrsim 300 \mathrm{pc}\right.$ ) of the molecular emission have $\mathrm{HCN} / \mathrm{HCO}^{+} \approx 1$. Thus, it appears that the more extended molecular emission is consistent with typical PDR environments in star-forming galaxies.

\subsection{The Origin and Fate of the Dense Molecular Wind}

Will this dense outflow escape the Galaxy or is it doomed to return to the nucleus? Using the dynamical mass obtained in Section 5 for the inner kpc, the escape velocity at $1 \mathrm{kpc}$ is $(G M / R)^{0.5} \sim 600 \mathrm{~km} \mathrm{~s}^{-1}$. The outflow velocities we see in the dense molecular gas reach a maximum of $\sim 300 \mathrm{~km} \mathrm{~s}^{-1}$ (Figure 5). Thus, it appears that these winds will be unable to escape the nucleus unless the outflow axis is highly inclined along the line of sight $\left(\theta \lesssim 35^{\circ}\right)$, reducing the projected radial velocity component. This observed wind, or at least its dense molecular component, appears destined to stall and remain bound to the system.

Dense gas conversion factors have been determined for "normal" galaxies, under the assumption that the emission is coming from an ensemble of distinct clouds (Gao \& Solomon 2004b). Estimating the mass outflow rate of dense material in IRAS 13120-5453 is problematic given the uncertain excitation and abundance of these dense gas tracers, as discussed above. Additionally, the ensemble of clouds assumption may not be appropriate for the nuclear ISM in ULIRGs, where the molecular gas may have a more smooth distribution (e.g., Solomon et al. 1997; Scoville et al. 2014).

However, we can provide an upper limit to the dense mass outflow rate, by using the ensemble of clouds approximation. If we assume the emission is optically thick and thermalized up to the 4-3 line, the equivalent $1-0$ luminosity of the outflow will equal that of the 4-3 line: $L_{\mathrm{HCN}(1-0)}^{\prime}=L_{\mathrm{HCN}(4-3)}^{\prime}=2 \times 10^{7} \mathrm{~K} \mathrm{~km} \mathrm{~s}^{-1} \mathrm{pc}^{2}$. This would correspond to a dense molecular mass of $2 \times 10^{8} M_{\odot}$ in the outflow.

Taking this upper limit on the outflowing dense gas mass we can compute the mass outflow rate, following Equation (1) of Cicone et al. (2014) and using the measured outflow extent of $r=0.2 \mathrm{kpc}$ and outflow velocity of $250 \mathrm{~km} \mathrm{~s}^{-1}$. We obtain an upper limit on the mass of outflowing dense molecular gas of $\dot{M}_{\text {dense }}<770 M_{\odot} \mathrm{yr}^{-1}$, significantly in excess of the $170 M_{\odot} \mathrm{yr}^{-1}$ SFR. Because the outflow appears to follow the rotation pattern (but lie above the flattening of the rotation curve), the velocity of the material identified as outflowing may have an important rotational component to its velocity, which would reduce the inferred $\dot{M}$.

We emphasize here that the above calculations merely represent an upper limit to the mass of dense gas detected here and the actual mass could be at least order of magnitude lower (e.g., Aalto et al. 2015b). That the outflowing gas shows a high $\mathrm{HCN} / \mathrm{HCO}^{+}$ratio $(>3)$ is strong evidence that the excitation and/or abundance is non-standard and that galactic conversion factors are unlikely to be accurate. An example of this is
Mrk 231, where the $\mathrm{HCN}, \mathrm{HCO}^{+}$, and $\mathrm{HNC}$ species do not appear to be co-spatial, but instead reflect an outflow with significant abundance variations in the wind (Lindberg et al. 2016), suggesting significant chemical effects which need to be considered for the appropriate interpretation of these molecules in outflows.

Within the substantial uncertainties in our mass outflow rate and the important consideration that we are only diagnosing the dense component of the outflow, the observations are broadly consistent with either an AGN or starburst-driven wind. CO observations (K. Sliwa 2016, in preparation) will be needed to both confirm the molecular outflow, estimate the total molecular outflow rate, and the dense fraction.

The outflow kinematics relative to the disk may shed some light on the origin of the outflow. A starburst-driven outflow would be expected to exit perpendicular to the disk (e.g., García-Burillo et al. 2001; Walter et al. 2002; Bolatto et al. 2013a), as the path of least resistance. In this case, there should be a $\sim 90^{\circ}$ shift in the PA of the disk, compared to the PA of the outflow. The HCN line wings in IRAS 13120-5453 have a similar PA to that of the disk (Figure 5) suggesting the wind is not exiting perpendicular to the disk. However, AGN outflows may be more collimated by the small-scale accretion disk and/ or torus. This small-scale collimation may have a random orientation with respect to the kpc-scale disk, so an AGNdriven outflow may be a more natural explanation for the wind identified here.

IRAS 13120-5453 was detected in $\mathrm{OH} 119 \mu \mathrm{m}$ absorption using Herschel observations (Veilleux et al. 2013), with maximum outflow velocities of up to $1200 \mathrm{~km} \mathrm{~s}^{-1}$. The $\mathrm{OH}$ outflow extends to significantly higher velocities than seen in the ALMA data. It is unclear if the outflow in this system is similar to the multi-phase outflow seen in Mrk 231 (Aalto et al. 2015b), where the HCN outflow corresponds to the lowervelocity $\mathrm{OH}$ outflow component, while the higher-velocity $\mathrm{OH}$ outflow does not have a corresponding HCN outflow. The velocity difference between $\mathrm{OH}$ and $\mathrm{HCN}$ outflow is broadly consistent with a fast, diffuse outflow (seen in $\mathrm{OH}$ ) that is entraining dense clouds at lower velocity.

HCN (4-3) emission associated with the lower-velocity portion of a CO-detected molecular outflow has been detected in Mrk 231 (Aalto et al. 2012a, 2015b), NGC 1068 (GarcíaBurillo et al. 2014), and M51 (Matsushita et al. 2015). The outflows are thought to be jet-driven (M51) or AGN-driven (Mrk 231, NGC 1068) based on the energetics or the inferred mass outflow rates. In these cases and in IRAS 13120-5453, the material identified as outflowing has a high $\mathrm{HCN} / \mathrm{HCO}^{+}$ ratio and is consistent with expectations from mechanical heating (e.g., Matsushita et al. 2015; Lindberg et al. 2016).

\subsection{The Relationship between $\Sigma_{I R}$ and $\Sigma_{14}$}

Based on the sizes measured from the submillimeter continuum, we have inferred a high $\Sigma_{\mathrm{IR}}$, indicating the presence of a fairly extreme starburst. In contrast, computing $\Sigma_{14}$ using the same size, we find a somewhat unremarkable luminosity surface density, relative to sources which feature IR pumping of $\mathrm{HCN}$.

Some insight into the apparent discrepancy can be gained by examining the $\Sigma_{\mathrm{IR}}$ and $\Sigma_{14}$ for an idealized singlecomponent blackbody. In particular, both quantities have different dependence on the temperature: $\Sigma_{\mathrm{IR}} \propto T^{4}$, and $\Sigma_{14} \propto\left(e^{h(21.4 \mathrm{THz}) /\left(k_{b} T\right)}-1\right)^{-1}$. In Figure 11 we show the 

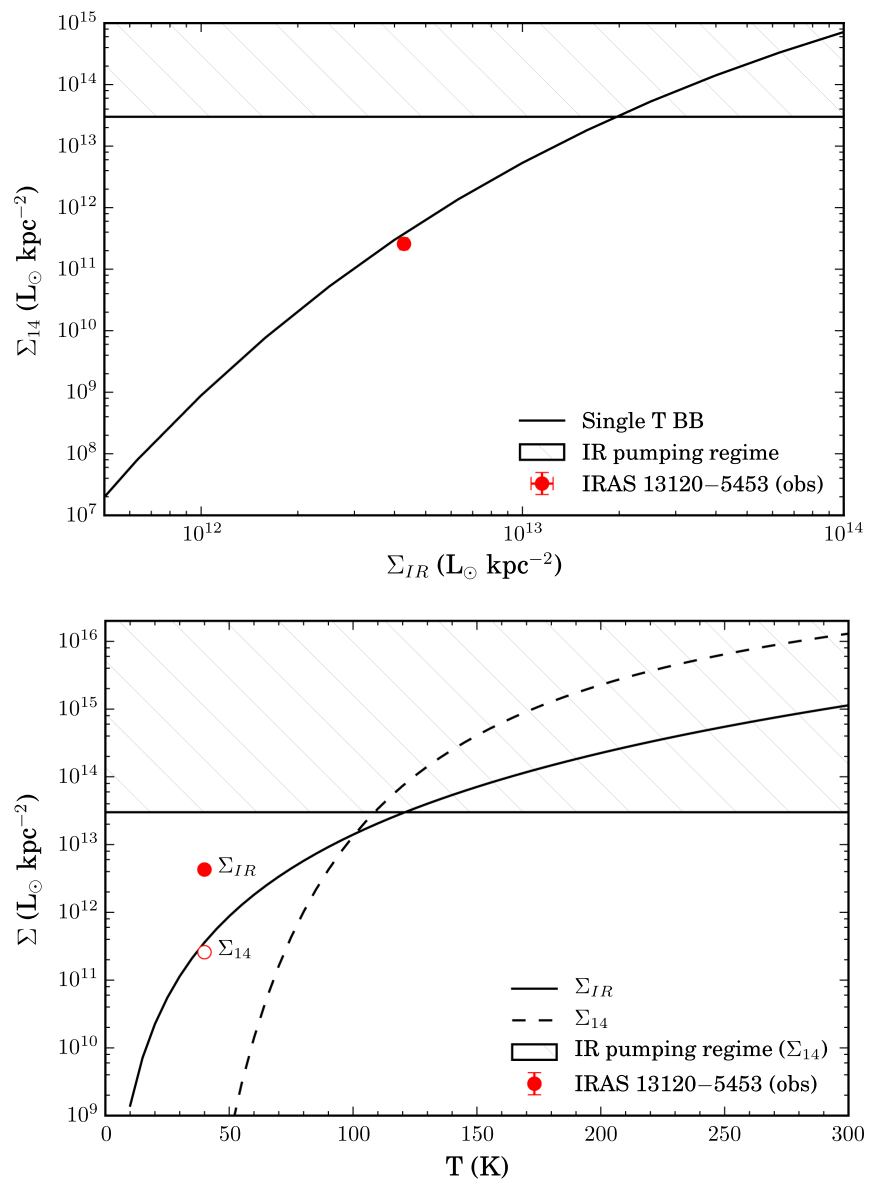

Figure 11. Top: the relationship between $\Sigma_{\mathrm{IR}}$ and $\Sigma_{14}$ for a single component blackbody. The temperature increases as a function of $\Sigma_{\mathrm{IR}}$ and $\Sigma_{14}$. The red point marks the observed $\Sigma_{\mathrm{IR}}$ and $\Sigma_{14}$ values for IRAS 13120-5453. The error bars are smaller than the point size. Bottom: explicit dependence of $\Sigma_{\mathrm{IR}}$ and $\Sigma_{14}$ on $T$ for a single component blackbody. For reference, $B_{\nu}(\nu, T)$ peaks at $\lambda_{\text {peak }}=14 \mu \mathrm{m}(21.4 \mathrm{THz})$ when $T=365 \mathrm{~K}$. In both figures, the hatched region marks where $\Sigma_{14}$ is high enough to potentially drive radiative pumping of $\mathrm{HCN}$ (note that pumping also requires sufficiently high column densities; Aalto et al. 2015a). For $T=40-100 \mathrm{~K}, \Sigma_{\mathrm{IR}}$ could be consistent with radiation pressure limited starbursts (modulo gas fraction and velocity dispersion; Thompson et al. 2005) without $\Sigma_{14}$ being high enough to support radiative pumping of HCN. The observed $\Sigma$ values for IRAS 13120-5453 (bottom panel) suggest the single temperature model is not a complete descriptor of the infrared-emitting region. On these scales, the error bars for the IRAS $13120-5453$ values are smaller than the point sizes.

relationship between $\Sigma_{\mathrm{IR}}$ and $\Sigma_{14}$ for a single-component blackbody, and the explicit dependence of these luminosity surface densities on the temperature. In the upper panel we plot the observed values for IRAS 13120-5453 and shade the range of $\Sigma_{14}$ values which may result in IR pumping of $\mathrm{HCN}$ (in the presence of sufficiently high column densities; Aalto et al. 2015a). Note that, in the top panel, there is an implicit dependence of $\Sigma_{\mathrm{IR}}$ and $\Sigma_{14}$ on $T$. While we do not explicitly plot $\Sigma_{13}$ (potentially relevant for the as-yet undetected IR pumping of $\mathrm{HCO}^{+}$) the curve closely tracks that of $\Sigma_{14}$.

These figures demonstrate that, for even fairly high $\Sigma_{\text {IR }}$ systems which could be radiation pressure limited starbursts (i.e., those between $10^{12}$ and $10^{13} L_{\odot} \mathrm{kpc}^{-2}$, with the exact value depending on $\sigma$ and $f_{\text {gas }}$; Thompson et al. 2005), $\Sigma_{14}$ can remain below the range required for radiative pumping of HCN. Thus, some systems with radiation pressure limited starbursts may not show vibrational HCN emission, unless they have an embedded compact core with $\Sigma_{14}>3 \times 10^{13}$ (corresponding to $\Sigma_{\mathrm{IR}} \gtrsim 2 \times 10^{13} L_{\odot} \mathrm{kpc}^{-2}$ for a singlecomponent blackbody).

\subsection{A Scenario for the Nuclear Activity in IRAS 13120-5453}

The overall properties of IRAS 13120-5453 are nearly consistent with the properties of these CONs, notably the presence of a Compton-thick AGN (Teng et al. 2015) and a significant [C II] deficit (Díaz-Santos et al. 2014). However, an important difference between IRAS 13120-5453 and the CONs is the lack of vibrational $\mathrm{HCN}$ emission and a substantially lower estimated $14 \mu \mathrm{m}$ luminosity surface density. Despite the high $\mathrm{HCN} / \mathrm{HCO}^{+}$ratio and the [C II] deficit, IRAS 13120-5453 does not seem to be as extreme as the systems presented by Aalto et al. (2015a). We note that while the $40 \mathrm{~K}$ dust temperature is higher than typical nearby galaxies, which have $T_{\text {dust }} \sim 25 \mathrm{~K}$ (e.g., Skibba et al. 2011), this is similar to dust temperatures in local IR-selected galaxies (R. HerreroIllana 2016, in preparation) and much cooler than is seen in CONs such as Arp 220, NGC 4418, Mrk 231, and Zw 049.057 $\left(T_{\text {dust }}=90-130,130-150,110\right.$, and $>100 \mathrm{~K}$, respectively; González-Alfonso et al. 2012, 2013, 2014; Falstad et al. 2015).

The elevated $\mathrm{HCN}(4-3) / \mathrm{HCO}^{+}(4-3)$ ratio is consistent with a $\mathrm{HCN}$ abundance enhancement from mechanical heating, implying a turbulent nuclear ISM. The outflows in this system suggest the feedback from star formation and/or the AGN is pushing the nuclear ISM to larger radii. One explanation for the lack of $v_{2}=1 f$ emission is that this feedback has sufficiently inflated the nuclear gas concentration to the point where the $14 \mu \mathrm{m}$ luminosity density is too low to drive IR pumping. Stated another way, the movement of gas (and associated dust) to larger distance from the central starburst may effectively reduce the dust temperature by reducing the optical depth to UV and IR photons.

If the CON phase is a universal (but likely short-lived) phase in a luminous merger, is IRAS $13120-5453$ in a pre- or postCON state? Given the advanced dynamical stage of the system (post-merger; Haan et al. 2011; Stierwalt et al. 2013) and the presence of loops, it is likely the nucleus will continue to receive a modest inflow of gas as the tidal material continues to re-accrete (e.g., as seen in NGC 7252; Hibbard \& Mihos 1995). However, it seems unlikely that the Galaxy will experience substantial large-scale tidal torques which would drive significant additional quantities of gas into the nucleus on a short timescale, such as what likely triggered the present starburst (e.g., Barnes \& Hernquist 1991). That the starburst appears compact, yet lacks significant $\mathrm{HCN} v_{2}=1 f$ emission, has a $\Sigma_{14 \mu \mathrm{m}}$ lower than CONs, and shows evidence for outflows, suggests (if IRAS 13120-5453 experienced a CON phase) we may be seeing IRAS $13120-5453$ in a post-CON phase where feedback is clearing the nucleus. This is in qualitative agreement with the results of Aalto et al. (2015a), finding that systems with the most luminous $v_{2}=1 f$ lines (relative to $L_{\mathrm{IR}}$ ) lack outflows, and suggests a CON phase precedes the onset of significant outflows. If the feedback is starburst-driven, this may imply future starburst episodes as the dense gas wind stalls and returns (e.g., Torrey et al. 2016).

One remaining question regarding the outflow concerns the $\sim 1200 \mathrm{~km} \mathrm{~s}^{-1}$ outflow seen in $\mathrm{OH}$ (Veilleux et al. 2013): does this fast component of the outflow contain a significant amount of mass which will become unbound from the system? If the 
$\mathrm{OH}$ outflow represents only the tail of the distribution of outflowing material, the bulk of the ISM will remain bound. What fraction of the ISM is escaping will impact whether the feedback is regulating or quenching star formation. Given $\sim 30 \%$ of the dynamical mass in the central kpc is currently gas, determining whether that gas is permanently removed from the system has significant implications for the structure of the remnant galaxy and the growth of the central SMBH.

\section{Conclusions}

We present ALMA Band 7 observations of $\mathrm{HCN}(4-3)$ $\left(v=0\right.$ and $\left.v_{2}=1 f\right), \mathrm{HCO}^{+}(4-3), \mathrm{CS}(7-6)$, and the $333 \mathrm{GHz}$ continuum, and Herschel observations of $\mathrm{H}_{2} \mathrm{O}$ lines. We do not detect the $v_{2}=1 f$ line of $\mathrm{HCN}$ and the ratio of the $v=0$ / $v_{2}=1 f$ lines is $>10 \times$ higher than seen in sources with detectable IR pumping of HCN. Using these data, we show:

1. The $\mathrm{HCN} / \mathrm{HCO}^{+}$ratio is elevated over the nucleus and is consistent with the expectations of increased abundance due to mechanical heating by supernovae, though the AGN and the outflows may contribute additional turbulent energy.

2. Line wings on the $\mathrm{HCN}$ and $\mathrm{HCO}^{+}$profiles provide evidence for the presence of a dense molecular outflow that exceeds the virial range for the gas disk but will remain bound to the system. The large uncertainties in the abundance and excitation of HCN hamper estimates of the mass and means we cannot distinguish between an AGN or starburst origin based on energetics.

3. Our modeling of the $\mathrm{H}_{2} \mathrm{O}$ emission suggests a nuclear ISM that has a moderate dust temperature $(40 \mathrm{~K})$, higher than normal galaxies but a factor of $>2$ cooler than $T_{\text {dust }}$ inferred for CONs.

4. We marginally resolve the $333 \mathrm{GHz}$ continuum emission, which implies a starburst diameter of $\sim 500 \mathrm{pc}$ and an infrared surface density of $\Sigma_{\mathrm{IR}}=4.7 \times 10^{12} L_{\odot} \mathrm{kpc}^{-2}$. This is near to, but below predictions from radiation pressure limited starburst models.

5. A potentially large fraction of the dynamical mass within the central kpc is gaseous, making the fate of this nuclear gas critical to predicting the long-term evolution of the structure of IRAS 13120-5453 and the growth of its central SMBH.

Combining this evidence, we propose that IRAS 13120-5453 is being observed during a period of nuclear activity where feedback is inflating the nuclear gas configuration and restricting star formation. The dense molecular outflow will likely not escape the system and so will return to the nucleus to fuel a future episode of star formation and SMBH accretion. The observed $\mathrm{HCN} / \mathrm{HCO}^{+}$ratio is consistent with mechanical energy injection from supernovae, though the AGN and outflows may also contribute.

The authors thank the anonymous referee for his/her comments, which have improved the quality of the paper. This paper makes use of the following ALMA data: ADS/JAO. ALMA\#2012.1.00817.S. ALMA is a partnership of ESO (representing its member states), NSF (USA) and NINS (Japan), together with NRC (Canada) and NSC and ASIAA (Taiwan) and KASI (Republic of Korea), in cooperation with the Republic of Chile. The Joint ALMA Observatory is operated by ESO, AUI/NRAO and NAOJ. The National Radio Astronomy
Observatory is a facility of the National Science Foundation operated under cooperative agreement by Associated Universities, Inc.

Herschel is an ESA space observatory with science instruments provided by European-led Principal Investigator consortia and with important participation from NASA.

G.C.P. was supported by a FONDECYT Postdoctoral Fellowship (No. 3150361) and acknowledges the hospitality of the National Socio-environmental Synthesis Center (SESYNC), where portions of this manuscript were written. G.C.P. and E.T. acknowledge support from the CONICYT Anillo project ACT1101. F.C. acknowledges support from Swedish National Science Council grant 637-2013-7261. S.G. B. acknowledges support from Spanish grants AYA201342227-P and ESP2015-68964-P. K.S. acknowledges grant 1052119-M-001-036 from Taiwanese Ministry of Science and Technology.

Portions of this work were performed at the Aspen Center for Physics, which is supported by National Science Foundation grant PHY-1066293. This work was partially supported by a grant from the Simons Foundation.

The authors thank Claudia Cicone, Francois Schweizer, and Claudio Ricci for comments on an earlier version of the manuscript. G.C.P. thanks Paul Torrey, David Patton, Chris Hayward, Desika Narayanan, Claudia Cicone, Nick Scoville, and Patricia Bessiere for helpful discussions.

This research has made use of the NASA/IPAC Extragalactic Database (NED) which is operated by the Jet Propulsion Laboratory, California Institute of Technology, under contract with the National Aeronautics and Space Administration. This research has made use of NASA's Astrophysics Data System. This research made use of ipython (Pérez \& Granger 2007), numpy (Van Der Walt et al. 2011), Astropy (Astropy Collaboration et al. 2013, http://www. astropy.org, a community-developed core Python package for Astronomy), emcee (Foreman-Mackey et al. 2013), and the dust_emissivity package (https://github.com/keflavich/ dust_emissivity). The figures in this paper were created using matplotlib (Hunter 2007) and APLpy (Robitaille \& Bressert 2012, an open-source plotting package for Python hosted at http://aplpy.github.com).

\section{References}

Aalto, S., Booth, R. S., Black, J. H., \& Johansson, L. E. B. 1995, A\&A, 300,369

Aalto, S., Costagliola, S. M. F., Gonzalez-Alfonso, E., et al. 2015a, A\&A, 584, A42

Aalto, S., Garcia-Burillo, S., Muller, S., et al. 2012a, A\&A, 537, A44

Aalto, S., Garcia-Burillo, S., Muller, S., et al. 2015b, A\&A, 574, A85

Aalto, S., Muller, S., Sakamoto, K., et al. 2012b, A\&A, 546, A68

Alatalo, K., Blitz, L., Young, L. M., et al. 2011, ApJ, 735, 88

Armus, L., Mazzarella, J. M., Evans, A. S., et al. 2009, PASP, 121, 559

Astropy Collaboration, Robitaille, T. P., Tollerud, E. J., et al. 2013, A\&A, 558, A33

Barnes, J. E., \& Hernquist, L. E. 1991, ApJL, 370, L65

Bianchi, S. 2013, A\&A, 552, A89

Bigiel, F., Leroy, A., Walter, F., et al. 2008, AJ, 136, 2846

Bolatto, A. D., Warren, S. R., Leroy, A. K., et al. 2013a, Natur, 499, 450

Bolatto, A. D., Wolfire, M., \& Leroy, A. K. 2013b, ARA\&A, 51, 207

Bouché, N., Carfantan, H., Schroetter, I., Michel-Dansac, L., \& Contini, T. 2015, AJ, 150, 92

Briggs, D. 1995, PhD thesis, The New Mexico Institute of Mining and Technology

Carroll, T. J., \& Goldsmith, P. F. 1981, ApJ, 245, 891

Cicone, C., Maiolino, R., Sturm, E., et al. 2014, A\&A, 562, A21

Costagliola, F., Aalto, S., Rodriguez, M. I., et al. 2011, A\&A, 528, A30 
Costagliola, F., Aalto, S., Sakamoto, K., et al. 2013, A\&A, 556, A66

Cruddace, R., Paresce, F., Bowyer, S., \& Lampton, M. 1974, ApJ, 187, 497

Daniel, F., Dubernet, M.-L., \& Grosjean, A. 2011, A\&A, 536, A76

Davies, R. I., Mark, D., \& Sternberg, A. 2012, A\&A, 537, A133

Díaz-Santos, T., Armus, L., Charmandaris, V., et al. 2013, ApJ, 774, 68

Díaz-Santos, T., Armus, L., Charmandaris, V., et al. 2014, ApJL, 788, L17

Díaz-Santos, T., Charmandaris, V., Armus, L., et al. 2010, ApJ, 723, 993

Downes, D., \& Eckart, A. 2007, A\&A, 468, L57

Dubernet, M.-L., Daniel, F., Grosjean, A., \& Lin, C. Y. 2009, A\&A, 497, 911

Falstad, N., González-Alfonso, E., Aalto, S., et al. 2015, A\&A, 580, A52

Feruglio, C., Fiore, F., Piconcelli, E., et al. 2013, A\&A, 558, A87

Feruglio, C., Maiolino, R., Piconcelli, E., et al. 2010, A\&A, 518, L155

Fischer, J., Luhman, M. L., Satyapal, S., et al. 1999, Ap\&SS, 266, 91

Fischer, J., Sturm, E., González-Alfonso, E., et al. 2010, A\&A, 518, L41

Foreman-Mackey, D., Hogg, D. W., Lang, D., \& Goodman, J. 2013, PASP, 125,306

Gao, Y., \& Solomon, P. M. 2004a, ApJ, 606, 271

Gao, Y., \& Solomon, P. M. 2004b, ApJS, 152, 63

García-Burillo, S., Combes, F., Usero, A., et al. 2014, A\&A, 567, A125

García-Burillo, S., Combes, F., Usero, A., et al. 2015, A\&A, 580, A35

García-Burillo, S., Martín-Pintado, J., Fuente, A., \& Neri, R. 2001, ApJL, 563, L27

García-Burillo, S., Usero, A., Alonso-Herrero, A., et al. 2012, A\&A, 539, A8

González-Alfonso, E., \& Cernicharo, J. 1997, A\&A, 322, 938

González-Alfonso, E., \& Cernicharo, J. 1999, ApJ, 525, 845

González-Alfonso, E., Fischer, J., Aalto, S., \& Falstad, N. 2014, A\&A, 567, A91

González-Alfonso, E., Fischer, J., Bruderer, S., et al. 2013, A\&A, 550, A25

González-Alfonso, E., Fischer, J., Graciá-Carpio, J., et al. 2012, A\&A, 541, A4

González-Alfonso, E., Fischer, J., Sturm, E., et al. 2015, ApJ, 800, 69

González-Alfonso, E., Smith, H. A., Ashby, M. L. N., et al. 2008, ApJ, 675,303

González-Alfonso, E., Smith, H. A., Fischer, J., \& Cernicharo, J. 2004, ApJ, 613,247

González-Martín, O., Rodríguez-Espinosa, J. M., Díaz-Santos, T., et al. 2013, A\&A, 553, A35

Griffin, M. J., Abergel, A., Abreu, A., et al. 2010, A\&A, 518, L3

Haan, S., Surace, J. A., Armus, L., et al. 2011, AJ, 141, 100

Hibbard, J. E., \& Mihos, J. C. 1995, AJ, 110, 140

Hinshaw, G., Weiland, J. L., Hill, R. S., et al. 2009, ApJS, 180, 225

Hunter, J. D. 2007, CSE, 9, 90

Imanishi, M., \& Nakanishi, K. 2013, AJ, 146, 91

Imanishi, M., Nakanishi, K., \& Kohno, K. 2006, AJ, 131, 2888

Imanishi, M., Nakanishi, K., Tamura, Y., Oi, N., \& Kohno, K. 2007, AJ, 134, 2366

Inami, H., Armus, L., Charmandaris, V., et al. 2013, ApJ, 777, 156

Iwasawa, K., Mazzarella, J. M., Surace, J. A., et al. 2011, A\&A, 528, A137

Izumi, T., Kohno, K., Aalto, S., et al. 2015, ApJ, 811, 39

Izumi, T., Kohno, K., Aalto, S., et al. 2016, ApJ, 818, 42

Izumi, T., Kohno, K., Martín, S., et al. 2013, PASJ, 65, 100

Kamenetzky, J., Rangwala, N., Glenn, J., Maloney, P. R., \& Conley, A. 2016, ApJ, 829, 93

Kazandjian, M. V., Meijerink, R., Pelupessy, I., Israel, F. P., \& Spaans, M. 2012, A\&A, 542, A65

Kim, D.-C., Evans, A. S., Vavilkin, T., et al. 2013, ApJ, 768, 102

Kohno, K., Matsushita, S., Vila-Vilaró, B., et al. 2001, in ASP Conf. Ser. 249, The Central Kiloparsec of Starbursts and AGN: The La Palma Connection, ed. J. H. Knapen et al. (San Francisco, CA: ASP), 672

Krumholz, M. R. 2014, MNRAS, 437, 1662
Lepp, S., \& Dalgarno, A. 1996, A\&A, 306, L21

Lindberg, J. E., Aalto, S., Muller, S., et al. 2016, A\&A, 587, A15

Loenen, A. F., Spaans, M., Baan, W. A., \& Meijerink, R. 2008, A\&A, 488, L5

Matsushita, S., Trung, D.-V., Boone, F., et al. 2015, ApJ, 799, 26

Mattila, S., \& Meikle, W. P. S. 2001, MNRAS, 324, 325

McMullin, J. P., Waters, B., Schiebel, D., Young, W., \& Golap, K. 2007, in ASP Conf. Ser. 376, Astronomical Data Analysis Software and Systems XVI, ed. R. A. Shaw, F. Hill, \& D. J. Bell (San Francisco, CA: ASP), 127 Meijerink, R., Spaans, M., \& Israel, F. P. 2007, A\&A, 461, 793

Mills, E. A. C., Güsten, R., Requena-Torres, M. A., \& Morris, M. R. 2013, ApJ, 779, 47

Morrison, R., \& McCammon, D. 1983, ApJ, 270, 119

Mould, J. R., Huchra, J. P., Freedman, W. L., et al. 2000, ApJ, 529, 786

Ott, S. 2010, in ASP Conf. Ser. 434, Astronomical Data Analysis Software and Systems XIX, ed. Y. Mizumoto, K.-I. Morita, \& M. Ohishi (San Francisco, CA: ASP), 139

Pérez, F., \& Granger, B. E. 2007, CSE, 9, 21

Pickett, H. M., Poynter, R. L., Cohen, E. A., et al. 1998, JQSRT, 60, 883

Planck Collaboration, Abergel, A., Ade, P. A. R., et al. 2011, A\&A, 536, A25

Planck Collaboration, Ade, P. A. R., Aghanim, N., et al. 2014, A\&A, 571, A28

Poglitsch, A., Waelkens, C., Geis, N., et al. 2010, A\&A, 518, L2

Privon, G. C., Herrero-Illana, R., Evans, A. S., et al. 2015, ApJ, 814, 39

Rangwala, N., Maloney, P. R., Glenn, J., et al. 2011, ApJ, 743, 94

Requena-Torres, M. A., Güsten, R., Weiß, A., et al. 2012, A\&A, 542, L21

Robitaille, T., \& Bressert, E. 2012, APLpy: Astronomical Plotting Library in Python, Astrophysics Source Code Library, ascl:1208.017

Roche, P. F., \& Aitken, D. K. 1985, MNRAS, 215, 425

Roche, P. F., Alonso-Herrero, A., \& Gonzalez-Martin, O. 2015, MNRAS, 449, 2598

Sakamoto, K., Aalto, S., Combes, F., Evans, A., \& Peck, A. 2014, ApJ, 797, 90

Sakamoto, K., Aalto, S., Evans, A. S., Wiedner, M. C., \& Wilner, D. J. 2010, ApJL, 725, L228

Sakamoto, K., Scoville, N. Z., Yun, M. S., et al. 1999, ApJ, 514, 68

Scoville, N., Aussel, H., Sheth, K., et al. 2014, ApJ, 783, 84

Scoville, N., Sheth, K., Aussel, H., et al. 2016, ApJ, 820, 83

Scoville, N., Sheth, K., Walter, F., et al. 2015, ApJ, 800, 70

Scoville, N. Z., Yun, M. S., \& Bryant, P. M. 1997, ApJ, 484, 702

Skibba, R. A., Engelbracht, C. W., Dale, D., et al. 2011, ApJ, 738, 89

Solomon, P. M., Downes, D., \& Radford, S. J. E. 1992, ApJL, 387, L55

Solomon, P. M., Downes, D., Radford, S. J. E., \& Barrett, J. W. 1997, ApJ, 478,144

Stierwalt, S., Armus, L., Charmandaris, V., et al. 2014, ApJ, 790, 124

Stierwalt, S., Armus, L., Surace, J. A., et al. 2013, ApJS, 206, 1

Strauss, M. A., Huchra, J. P., Davis, M., et al. 1992, ApJS, 83, 29

Sturm, E., González-Alfonso, E., Veilleux, S., et al. 2011, ApJL, 733, L16

Teng, S. H., Rigby, J. R., Stern, D., et al. 2015, ApJ, 814, 56

Thompson, T. A., Quataert, E., \& Murray, N. 2005, ApJ, 630, 167

Torrey, P., Hopkins, P. F., Faucher-Giguère, C.-A., et al. 2016, arXiv:1601. 07186

van der Tak, F. F. S., Black, J. H., Schöier, F. L., Jansen, D. J., \& van Dishoeck, E. F. 2007, A\&A, 468, 627

Van Der Walt, S., Colbert, S. C., \& Varoquaux, G. 2011, arXiv:1102.1523

Veilleux, S., Meléndez, M., Sturm, E., et al. 2013, ApJ, 776, 27

Véron-Cetty, M.-P., \& Véron, P. 2001, A\&A, 374, 92

Walter, F., Weiss, A., \& Scoville, N. 2002, ApJL, 580, L21

Wilson, C. D., Petitpas, G. R., Iono, D., et al. 2008, ApJS, 178, 189

Zhang, Z.-Y., Gao, Y., Henkel, C., et al. 2014, ApJL, 784, L31

Ziurys, L. M., \& Turner, B. E. 1986, ApJL, 300, L19 